\title{
Novel Corrosion Inhibitors for Carbon Steel Alloy in Acidic Medium of 1N HCl Synthesized from Graphene Oxide
}

\author{
Hawraa H. Radey¹, Moayed N. Khalaf', Hadi Z. Al-Sawaad ${ }^{2}$ \\ ${ }^{1}$ Department of Chemistry, College of Science, University of Mysan, Basra, Iraq \\ ${ }^{2}$ Department of Chemistry, College of Science, University of Basrah, Basrah, Iraq \\ Email: mariumkarar@yahoo.com
}

How to cite this paper: Radey, H.H., Khalaf, M.N. and Al-Sawaad, H.Z. (2018) Novel Corrosion Inhibitors for Carbon Steel Alloy in Acidic Medium of $1 \mathrm{NHCl}$ Synthesized from Graphene Oxide. Open Journal of Organic Polymer Materials, 8, 53-79. https://doi.org/10.4236/ojopm.2018.84005

Received: September 17, 2018

Accepted: October 28, 2018

Published: October 31, 2018

Copyright $\odot 2018$ by authors and Scientific Research Publishing Inc. This work is licensed under the Creative Commons Attribution International License (CC BY 4.0).

http://creativecommons.org/licenses/by/4.0/

\begin{abstract}
In this study, two nano-derivatives from nano-Graphene oxide (GO) were synthesized. Regarding to GON and GOS by reaction GO with 2-amino ethanol and 2-marcapto ethanol respectively, the GO, GON, GOS were characterized by FTIR, XRD and FSEM. Evaluation prepared compound to inhibitors corrosion for Carbon steel in acidic media at ( $1-6 \mathrm{ppm})$ concentration and different temperature 298, 308, 318, $328 \mathrm{~K}$. The electrochemical technique used Tafel plot to measure the efficiency of inhibitor. It was observed that the corrosion rate and charge transfer of the carbon steel for the inhibitor increase with increase of temperature and decrease with increase of the inhibitor concentration in the same temperature. The GON had inhibition efficiency reached $96.96 \%$ for the $6 \mathrm{ppm}$ concentration at $298 \mathrm{~K}$.
\end{abstract}

\section{Keywords}

Graphene Oxide Derivatives, Corrosion Inhibitors, Carbon Steel Alloy

\section{Introduction}

Nowadays the nanocompounds are used in wide field of applications due to their ability to do them because of a large number of the functional groups in their chemical structures. One of them is graphene oxide (GO). In recent years, GO nanosheets have drawn special interest in various fields such as supercapacitors, batteries, and photocatalysis etc. The intriguing properties of GO arises from its chemical composition, which consists of graphene sheets with several oxygenated functional moieties attached, such as hydroxyl, carbonyl, carboxyl and epoxyl groups. In this respect, the chemical composition and physico-chemical 
properties of GO are attractive towards the application of corrosion resistant properties [1] [2]. The use of inhibitors for the control of corrosion for metals and alloys which are in contact with aggressive environment is an accepted practice. Large numbers of organic compounds were studied to investigate their corrosion inhibition potential. All these studies reveal that organic compounds especially those with N, S and O showed significant inhibition efficiency [3] [4]; inhibitors are added to the acidic solution during the acidizing process to reduce the aggressive corrosive effects of the acid on tubing and casing materials. Inhibitors are widely used for the protection of metals to corrosion in acidic environments. Inhibitors usually protect the metal by adsorbing on the surface and retarding metal corrosion in aggressive media. Therefore, selecting the appropriate inhibitor for a particular metal is very important. Most of the well-known inhibitors are organic compounds containing nitrogen, and oxygen [5]. A considerable number of studies have been published on the inhibition of steel and its alloys in acidic medium [6] [7] [8] [9] [10].

\section{Materials and Method}

\subsection{Materials}

C-steel (C1010) was obtained from Metal Samples (USA) was used with the following composition by percentage weight: $\mathrm{C}=0.13, \mathrm{Mn}=0.3, \mathrm{Si}=0.37, \mathrm{P}=$ $0.04, \mathrm{~S}=0.05, \mathrm{Cr}=0.1, \mathrm{Ni}=0.3, \mathrm{Cu}=0.3, \mathrm{AS}=0.08$ and the remainder is Fe. Ethanol amine, graphite, Hydrogen peroxide, Potassium permanganate, Sodium nitrate, maracapto ethanol from Fluka, Hydrochloric acid, Sulphuric acid from BDH.

\subsection{Experimental Methods}

\subsubsection{Graphene Oxide (GO)}

Graphene oxide was synthesized by Hummers method Graphite flakes (2 g) and $\mathrm{NaNO}_{3}(2 \mathrm{~g})$ were mixed in $50 \mathrm{~mL}$ of $\mathrm{H}_{2} \mathrm{SO}_{4}(98 \%)$ in a $1000 \mathrm{~mL}$ volumetric flask kept under at ice bath $\left(0^{\circ} \mathrm{C}-5^{\circ} \mathrm{C}\right)$ with continuous stirring. The mixture was stirred for $2 \mathrm{hrs}$ at this temperature and potassium permanganate $(6 \mathrm{~g})$ was added to the suspension very slowly. The rate of addition was carefully controlled to keep the reaction temperature lower than $15^{\circ} \mathrm{C}$. The ice bath was then removed, and the mixture was stirred at $35^{\circ} \mathrm{C}$ until it became pasty brownish and kept under stirring for one days. It is then diluted with slow addition of $100 \mathrm{ml}$ water. The reaction temperature was rapidly increased to $98^{\circ} \mathrm{C}$ with effervescence, and the color changed to brown color, Further this solution was diluted by adding additional $200 \mathrm{ml}$ of water stirred continuously for purification. The solution is finally treated with $10 \mathrm{ml} \mathrm{H}_{2} \mathrm{O}_{2}$ to terminate the reaction by appearance of yellow color, the mixture was washed by rinsing and centrifugation with $10 \% \mathrm{HCl}$ and then deionized (DI) water several times After filtration and drying under vacuum at room temperature (Scheme (1-1)) [11] [12] [13]. 


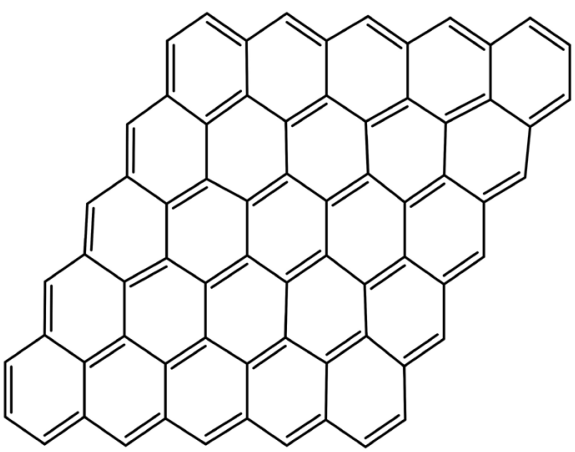

Graphite

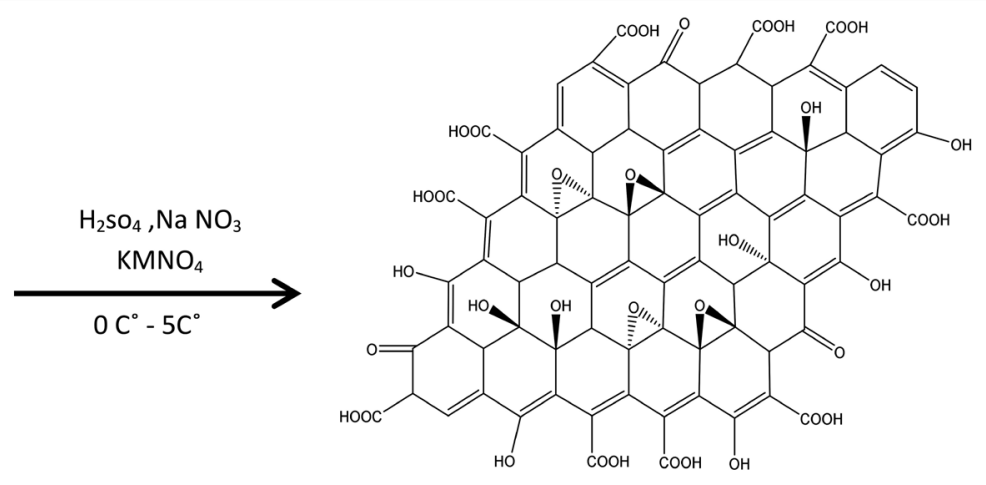

graphene oxide

Scheme (1-1). Chemical equation of preparation of GO.

\subsubsection{2-Amino Ethanol and Graphene Oxide Functionalized (GON)}

The functionalize GO, $(0.5 \mathrm{~g})$ was placed in a $(250 \mathrm{~mL})$ dried beaker with $(100 \mathrm{~mL})$ DMF and amino ethanol $6.17 \mathrm{~mL}, 10 \mathrm{mmole}$ ) sonicated for $1 \mathrm{~h}$ to form homogeneous solution, then added DCC (2.06 g, 10 mmole), DMAP (1.22 g, 10 mmole) into mixture with stir $24 \mathrm{~h}$ at room temperature, after the reaction finish, the product was filter, then black powder was dried [14] the preparation process of (GON) is shown in Scheme (2-2).

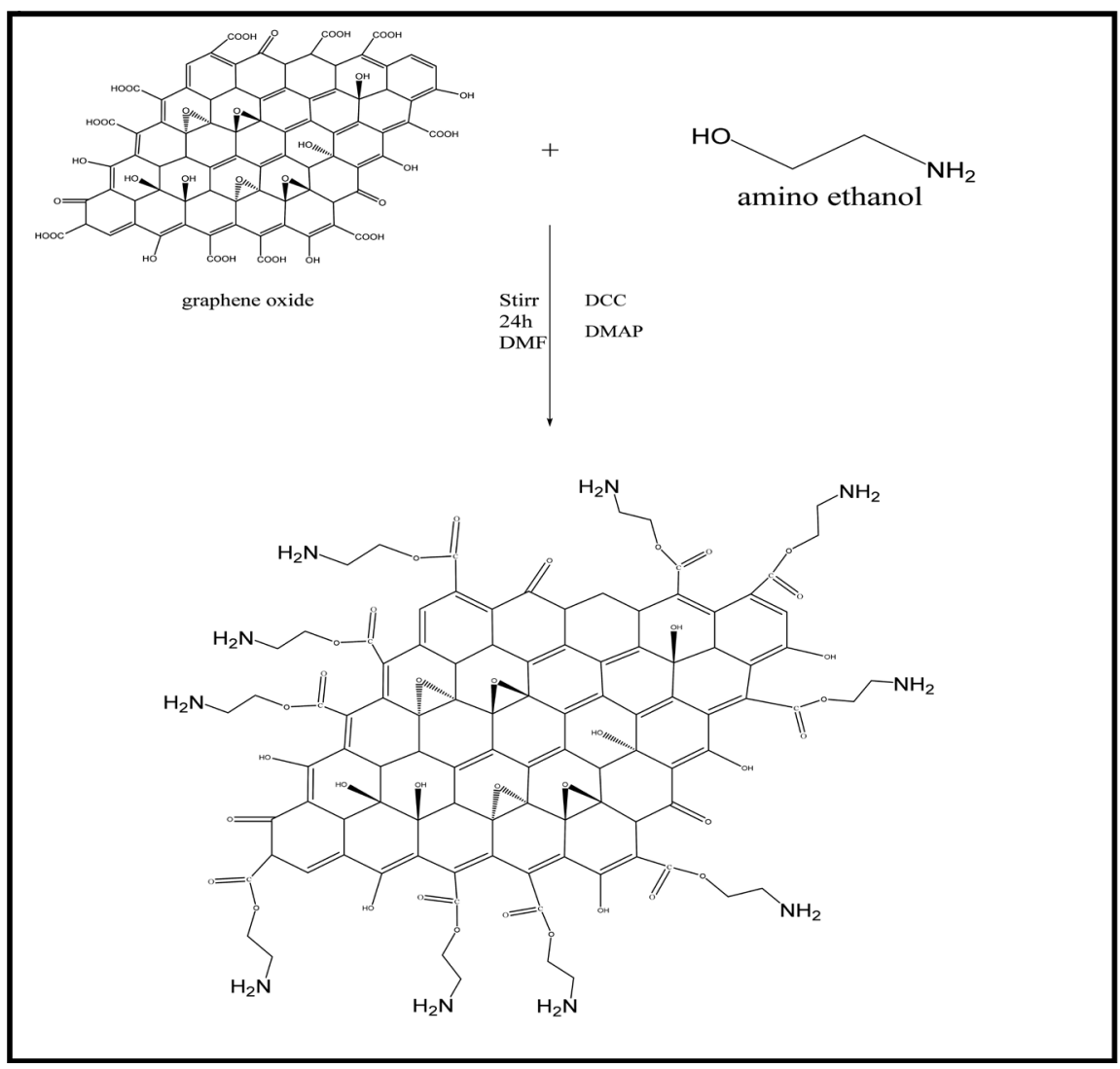

Scheme (2-2). Chemical equation of preparation (GON). 


\subsubsection{2-Mercapto Ethanol and Graphene Oxide Functionalized (GOS)}

The functionalize GO, $(0.5 \mathrm{~g})$ was placed in a $(250 \mathrm{~mL})$ dried beaker with $(100 \mathrm{~mL}) \mathrm{DMF}$ and amino ethanol $(0.86 \mathrm{~mL}, 10 \mathrm{mmole})$ sonicated for $1 \mathrm{~h}$ to form homogeneous solution, then added DCC (2.06 g, 10 mmole), DMAP $(1.22 \mathrm{~g}, 10 \mathrm{mmole})$ into mixture with stir $24 \mathrm{~h}$ at room temperature, after the reaction finish, the product was filter, then black powder was dried [14]. The preparation process of (GOS) is shown in Scheme (2-3).

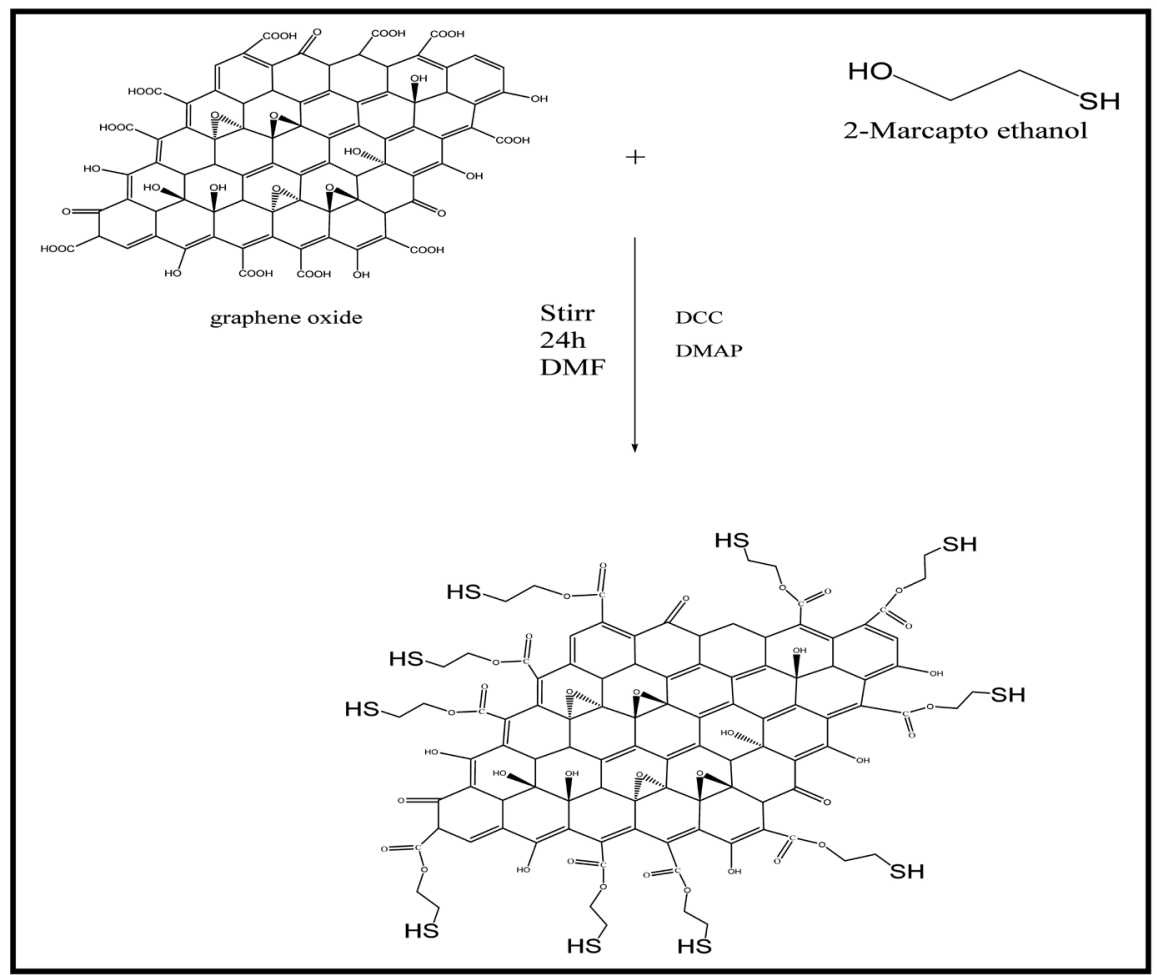

Scheme (2-3). Chemical equation of preparation (GOS).

\subsubsection{Electrochemical Measurements}

The electrochemical measurements were performed using a potentiostat/galvanostat (ACM) connected to a computer. A three electrode cell assembly, consisting of a C-steel rod embedded in araldite as the working electrode (WE), and a saturated calomel electrode as the reference electrode (RE), aplatinum sheet as the counter electrode (CE), was used for the electrochemical measurements. The temperature of the electrolyte was maintained at the required temperature using a water bath. Before immersion in the test solutions, the WE was polished with a polishing machine using emery paper from 600 to 1200 grade until a mirror image was obtained. Then, the WE was washed with distilled water thenimmersed in acetone for 1Minute in an ultrasonic cleaner. The WE electrode was prepared directly before electrochemical measurements then immersed in the test solution at open circuit potential for one hour until a steady state potential was obtained polarization measurements were performed. All experiments were performed in aerated solutions. From the pola- 
rization data, were calculated like the degree of surface coverage $(\theta)$, the percent tage inhibition efficiency (\% IE), corrosion rate and charge transfer resistance [15].

\section{Results and Discussion}

\subsection{FTIR Spectroscopy Analysis of GO, GON and GOS}

FTIR analysis was used to identify functional groups present in the GO, GON and GOS 1 . Figures 1 (a)-(c) shows the FTIR spectrum of, GO, GON 1 and $\mathrm{GOS}_{1}$ powders respectively, the GO spectrum shows the peak at $3386.39 \mathrm{~cm}^{-1}$ due to $\mathrm{O}-\mathrm{H}$ stretching vibration, $1724.36 \mathrm{~cm}^{-1}$ was strong $\mathrm{C}=\mathrm{O}$ stretching band, and peak at $16,228 \mathrm{~cm}^{-1}, 1378.85 \mathrm{~cm}^{-1}$ can be attributed to $\mathrm{C}=\mathrm{C}$ stretching of aromatic ring, $\mathrm{C}-\mathrm{OH}$ bending respectively, the charactestic peak at 1029.8 $\mathrm{cm}^{-1}$ due to C-O epoxy group [16] (Figure $1(\mathrm{~b})$ ). In the spectrum of GON the peak at $3434.6 \mathrm{~cm}^{-1}$ for stretching $\mathrm{OH}$ groups. The presence of absorption bands at $3334,3222 \mathrm{~cm}^{-1}\left(-\mathrm{NH}_{2}\right)$ stretching vibration, the peaks 2956,2823 $\mathrm{cm}^{-1}$ assigned to the asymmetric and symmetric stretching of $\mathrm{C}-\mathrm{H}$ bands, $1639.2 \mathrm{~cm}^{-1}$ due to $\mathrm{C}=\mathrm{O}$ aster stretching vibration, the peak at 1559.17, $1122,37 \mathrm{~cm}^{-1}$ attributed to $\mathrm{C}=\mathrm{C}$ aromatic ring, and $\mathrm{C}-\mathrm{N}$ stretching, also $1029.8 \mathrm{~cm}^{-1}$ due to $\mathrm{C}-\mathrm{O}$ epoxy group, the peak at $797.421 \mathrm{~cm}^{-1}$ due to aromatic C-H bending [17] (Figure 1(c)), GOS the peak at $3427.85 \mathrm{~cm}^{-1}$ for stretching $\mathrm{OH}$ groups. The peaks $2998,2898 \mathrm{~cm}^{-1}$ assigned to the asymmetric and symmetric stretching of $-\mathrm{CH}_{2}$ bands, also S-H band at $2550 \mathrm{~cm}^{1}, 1648.84$ $\mathrm{cm}^{-1}$ due to $\mathrm{C}=\mathrm{O}$ aster stretching vibration, the peak at 1559.77 is attributed to $\mathrm{C}=\mathrm{C}$ aromatic ring, a, also $1113.69,1024.02,620.966 \mathrm{~cm}^{-1}$ due to $\mathrm{C}-\mathrm{O}$, epoxy, C-S bending [17].

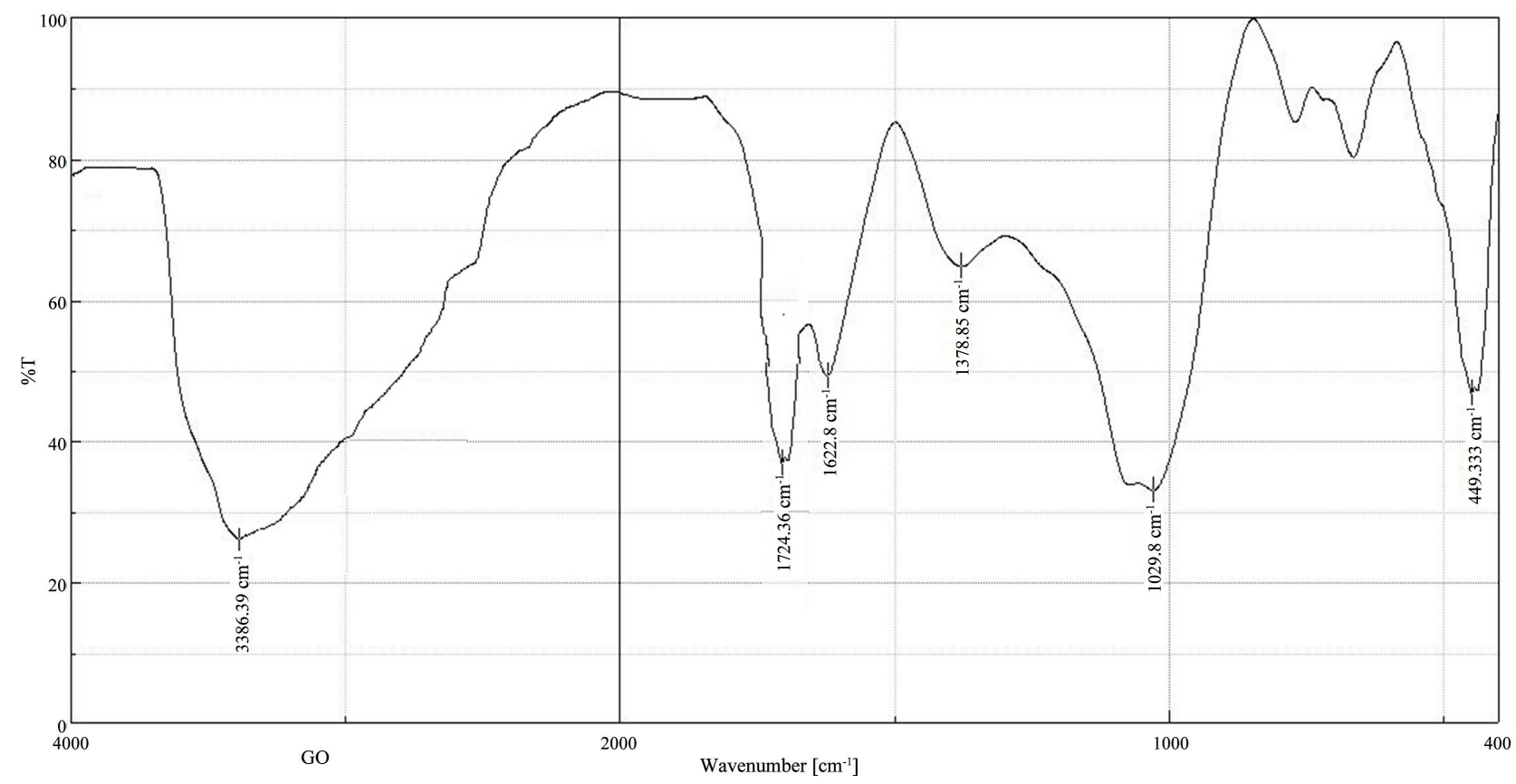

(a) 


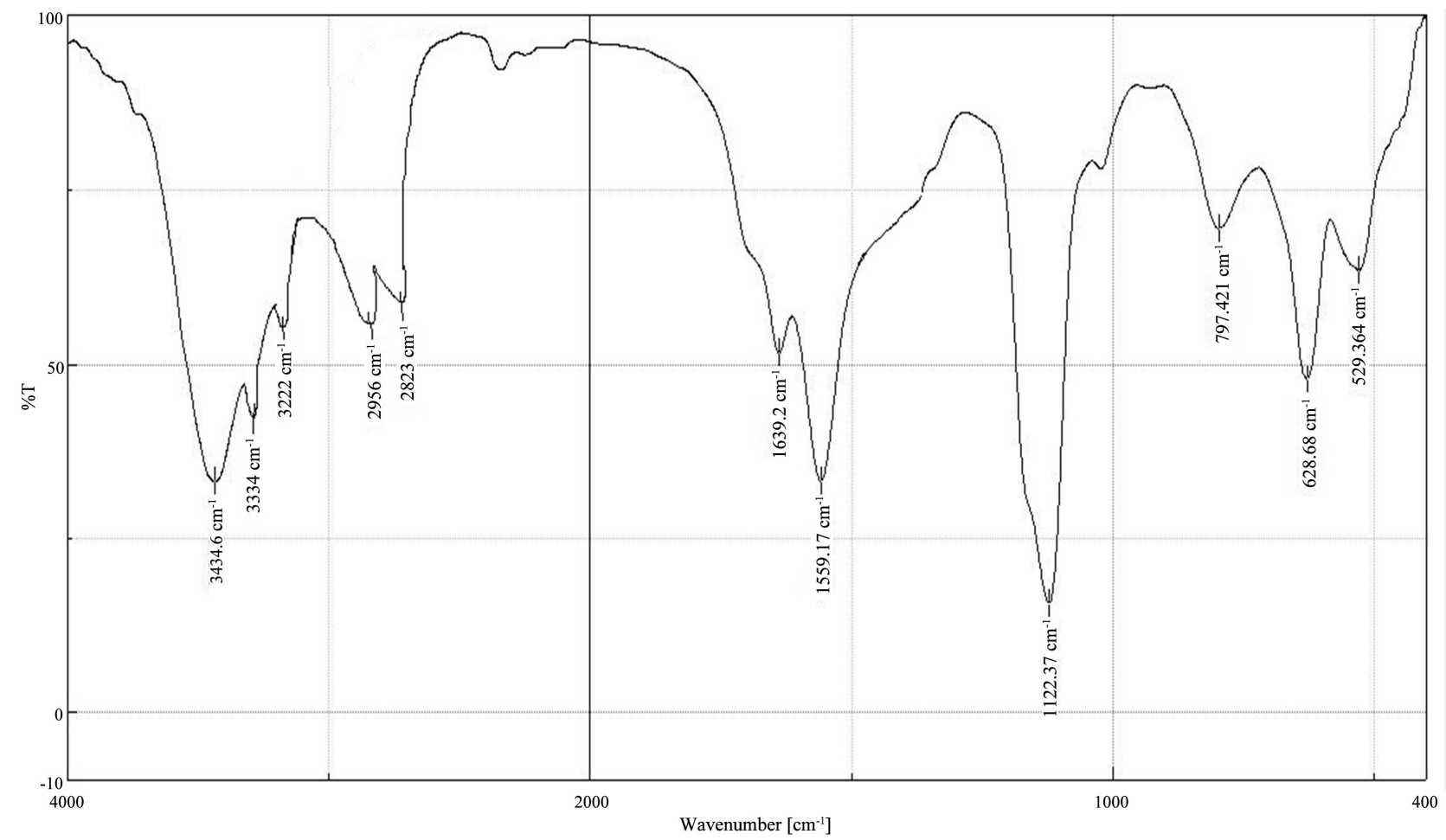

(b)

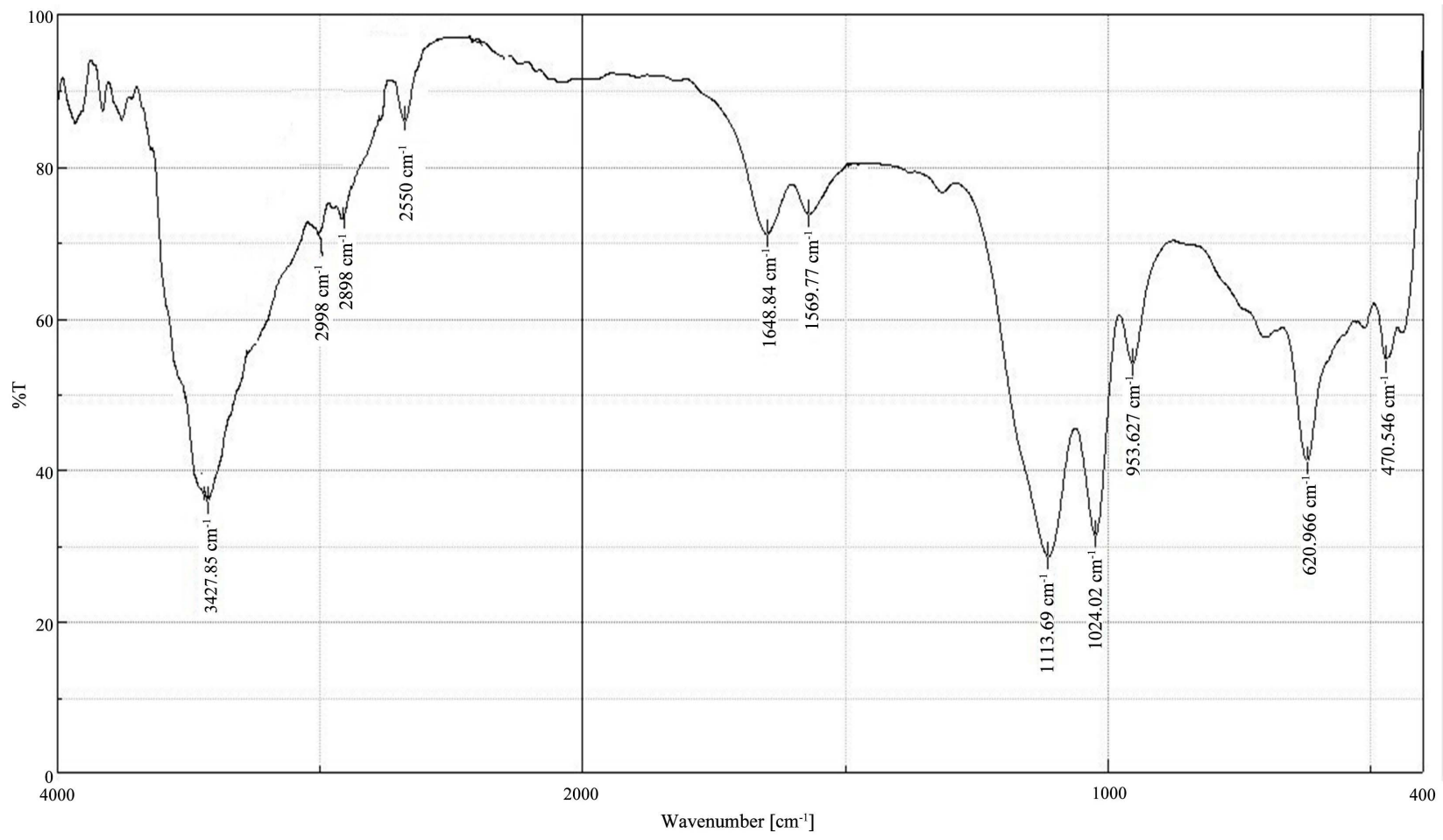

(c)

Figure 1. FTIR spectrum of (a) GO powder; (b) GON powder; (c) GOS powder.

\subsection{X-Ray Diffraction (XDR) of GO, GON and GOS}

In Figure 2(a) the X-Ray Diffraction (XRD) of grapheme oxide shows a large 
interlayer spacing equal to $8.06 \mathrm{~A}^{\circ}$ at the position $\left(2 \theta=10.97^{\circ}\right)$ disappearance of the peak at $26^{\circ}$ due to completely oxidized after the chemical oxidation and exfoliation [13] (Figure 2(b)), GON shows many peaks appear $(2 \theta=10.97)$ at d-spacing 8.06 indicate to graphene oxide and other peaks $2 \theta=34.05,38.80,54$, $72,55.35,58.43,62.15,65.24,67.64,71.28,72.55$ and 73.03 have to $d$-spacing $2.63,2.32,1.67,1.65,1.57,1.49,1.43,1.38 .1 .30$ and 1.29 , respectively attributed to functionalized ethanol amine at graphene oxide also intermediate layer [17], (Figure $2(\mathrm{c}))$ of GOS show many peaks appear $(2 \theta=10.97)$ at d-spacing 8.06 indicate to graphene oxide and other peaks $2 \theta=32.31,34.05,38.80,40.94,47.47$, $48.96,49,61,50.85,55.47,59.61,62.08,65.29$ and 67.63 have to d-spacing 2.77, $2.63,2.32,2.20,1.91,1.66,1.83,1.79 .1 .60,1.55,1.49,1.42$ and 1.38 , respectively attributed to functionalized 2-marcapto ethanol at graphene oxide also intermediate layer [17].

The Diffraction patterns of X-ray to prepared organic compound particles size is calculated using (Debye-Scherer) Equation [18]

$$
D=\frac{K \lambda}{\beta \operatorname{COS}_{\theta}}
$$

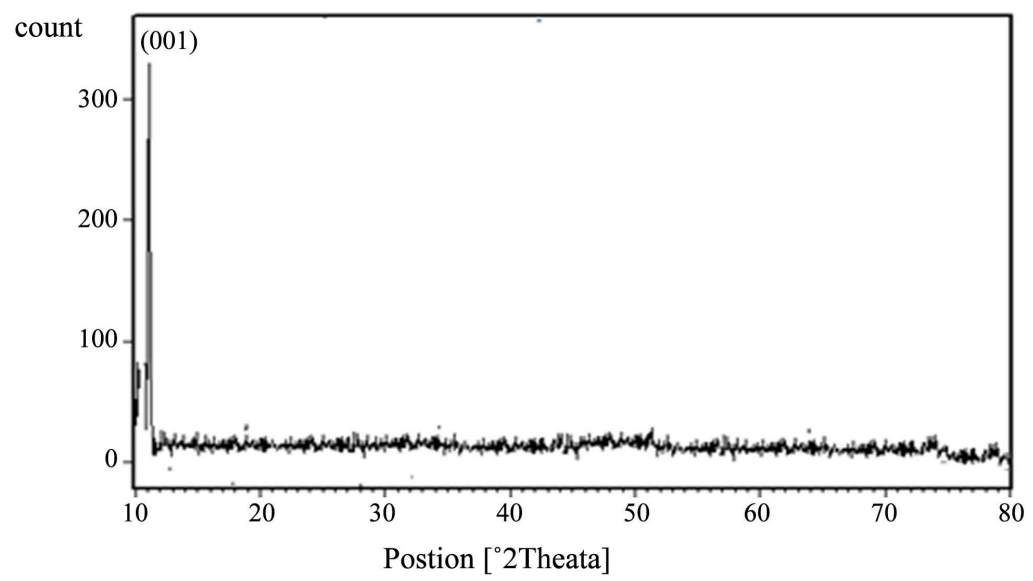

(a)

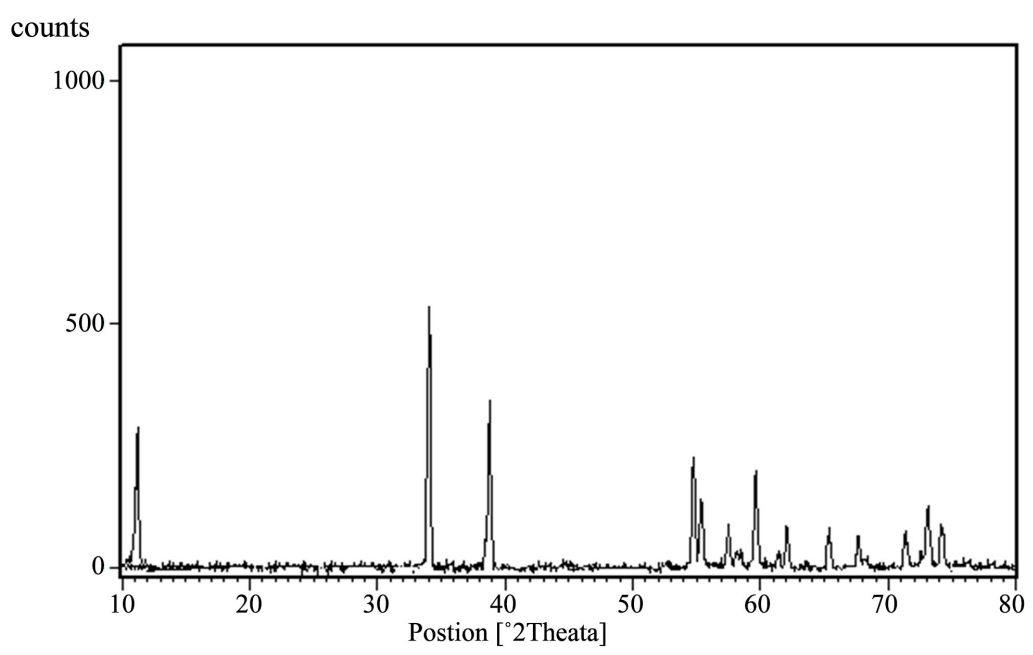

(b) 


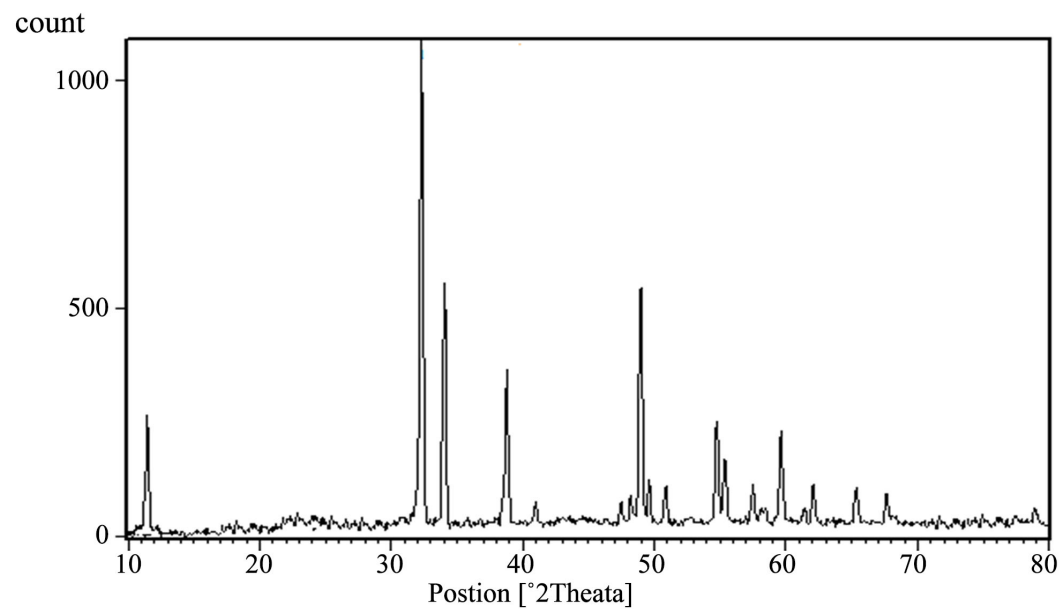

(c)

Figure 2. XRD OF (a) GO, (b) GON, (c) GOS.

where; $D$ : Particles size, $\lambda$ : X-ray wave length $(\mathrm{nm}), \beta$ : Half width at half maximum (HWHM), $K$ : is s related hape factor, normally taken as 0.9 . $\theta$ is x-ray angle. From this equation the particle size of grapheme oxide (GO) $(16 \mathrm{~nm})$ but to calculated average particle size to prepared organic compounds GON $(35.7 \mathrm{~nm})$, GOS (36.11 nm), Also calculatedparticles size using (Williamson-Hall) (W-H) equation [19]

$$
\beta * \cos \theta=\left[\frac{k \lambda}{D}\right]+[4 £+\operatorname{Sin} \theta]
$$

where $E$ micro strain of particles, the calculated particles size from graphic between $(\operatorname{Sin} \theta)$ on $\mathrm{x}$-axis, $(\beta * \cos \theta)$ on $\mathrm{y}$-axis, $D$ calculated by intercept $(K \lambda / D)$, Figure 3(a), from this equation particles size of GON $(17.74 \mathrm{~nm})$, micro strain (-0.00195), (b) GOS (14.26 nm) micro strain (-0.00246).

\subsection{Field Emission Scanning Electron Microscopy (FESEM)}

The FESEM of graphene oxide (GO) very sharp edges and flat surface the dark gray areas consist of several layers of sheets also kinked and wrinkled areas [13], shown in Figure 4(a) but GON observed very thin $(3.07 \mathrm{~nm})$ and re-stacked sheets (Figure 4(b)), GOSthe re-stacked layers and crumpling, kinked and wrinkled areas [20] [21] (Figure 4(c)).

\subsection{Electrochemical Measurements}

\subsubsection{Polarization Measurements (Tafel Method)}

Typical potentiodynamic polarization curves for the C-steel in $1 \mathrm{M} \mathrm{HCl}$ in the presence and absence of different concentrations of GO, GON, GOS are shown in (Figures 5-16). The respective Tafel parameters, inhibition efficiency (\% IE), $\mathrm{I}_{\text {corr }}$ corrosion current, corrosion rate and charge transfer resistance are provided in Tables 1-3. It is clear that the shapes of the Tafel plots for the inhibited electrodes are different from those of uninhibited electrodes. The presence of the in- 
hibitor decreases the current density but does not change other aspects of the behavior.

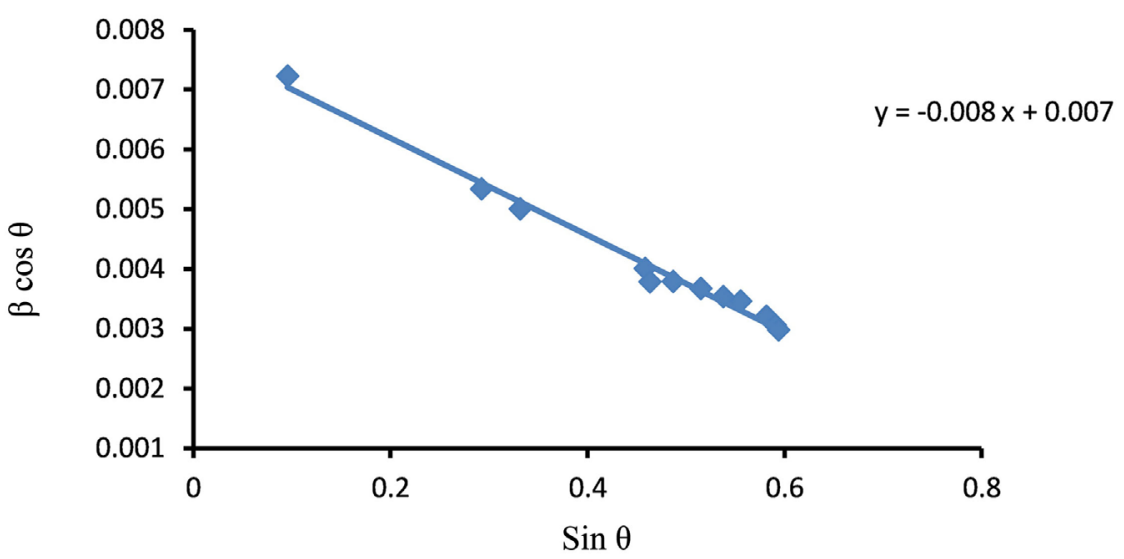

(a)

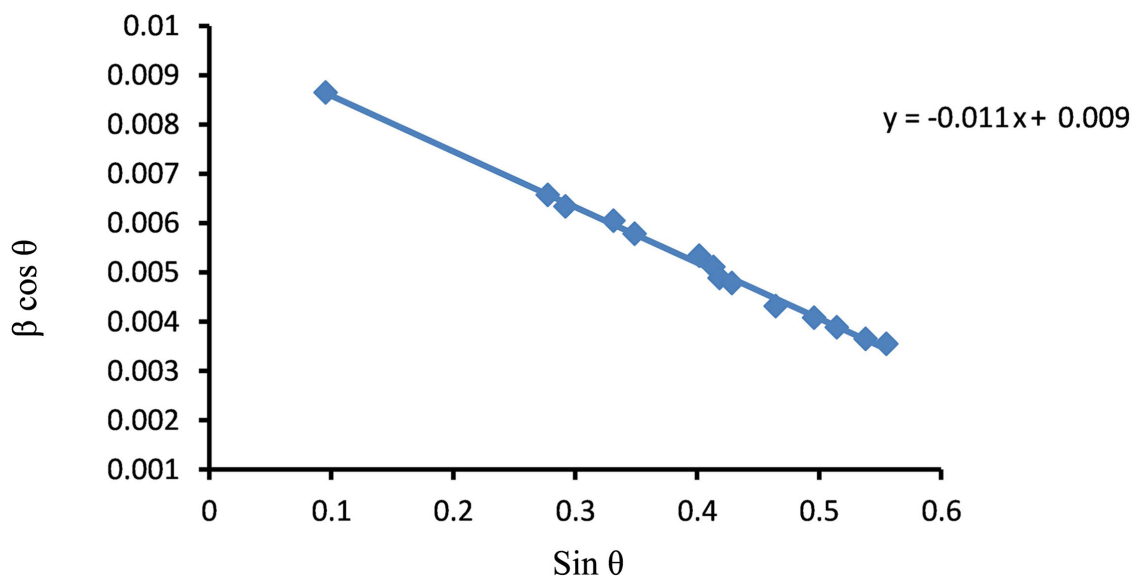

(b)

Figure 3. Scheme Williamson-Hall to (a) GON, (b) GOS.

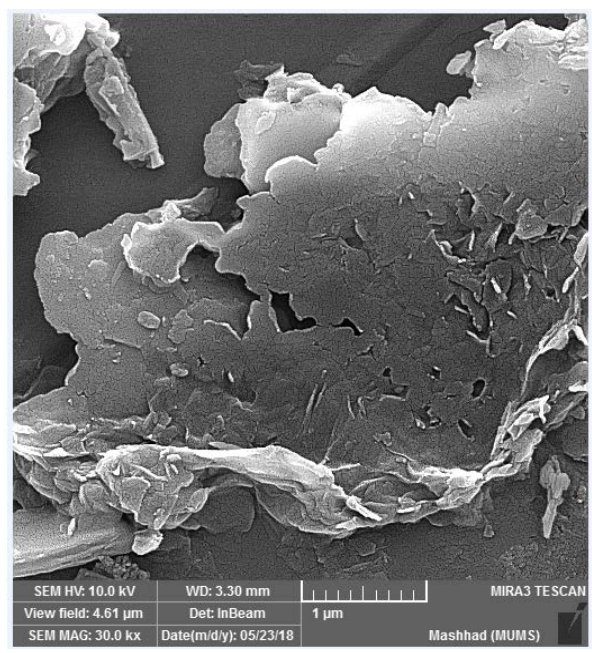

(a)

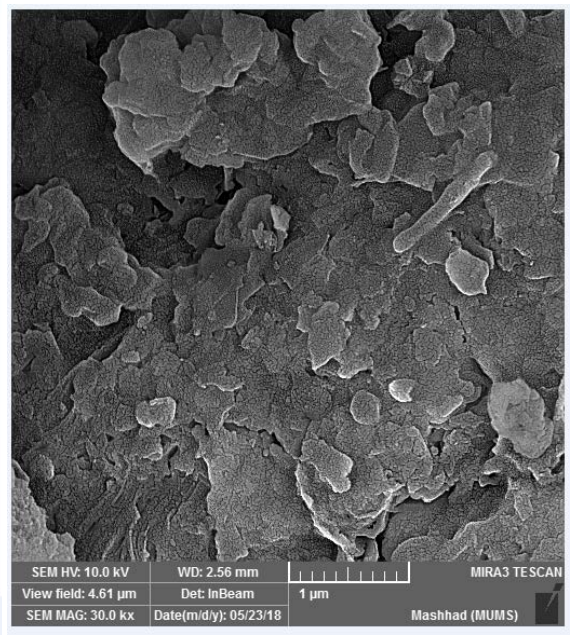

(b)

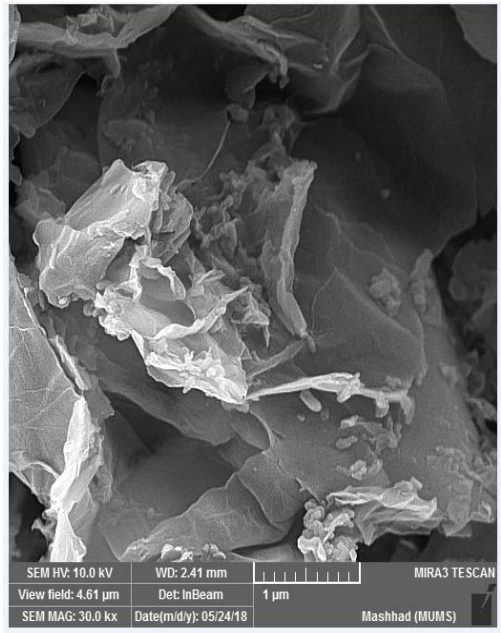

(c)

Figure 4. FESEM OF (a) GO, (b) GON, (c) GOS. 


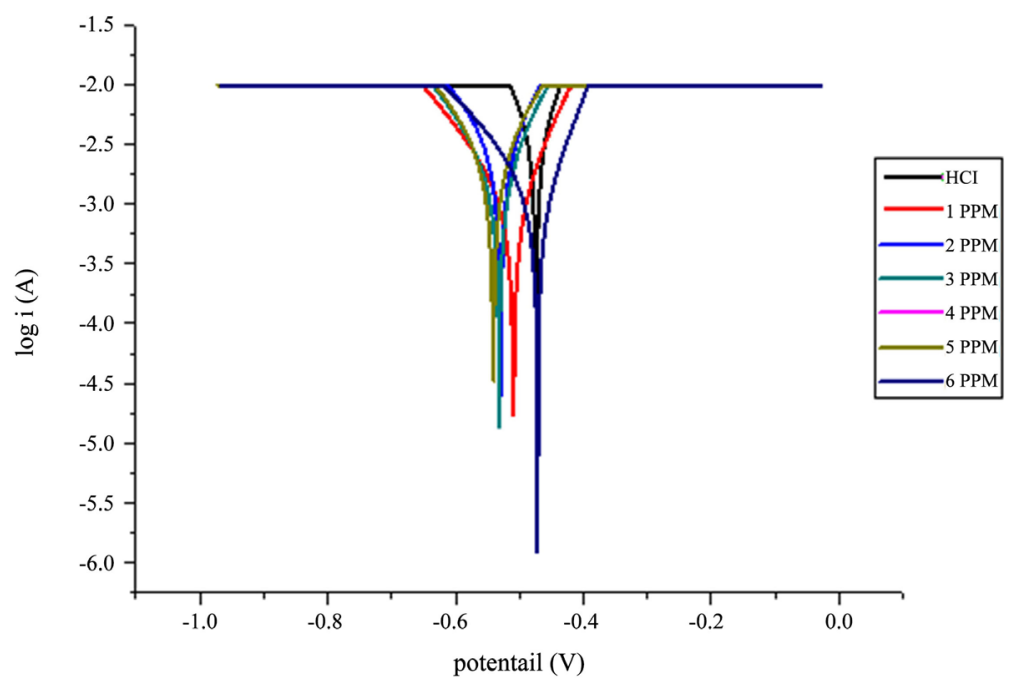

Figure 5. Tafel Plot of GOt concentration (1 - 6 ppm) on C-steel alloy in $298 \mathrm{k}$.

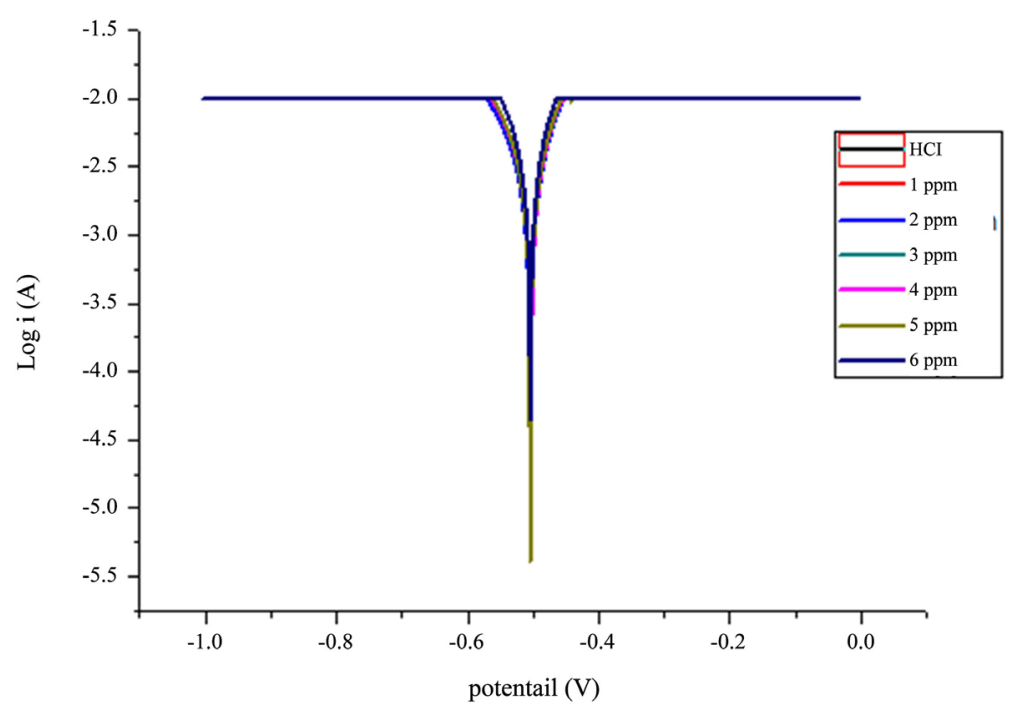

Figure 6. Tafel Plot of GO concentration (1 - 6 ppm) on C-steel alloy in $308 \mathrm{k}$.

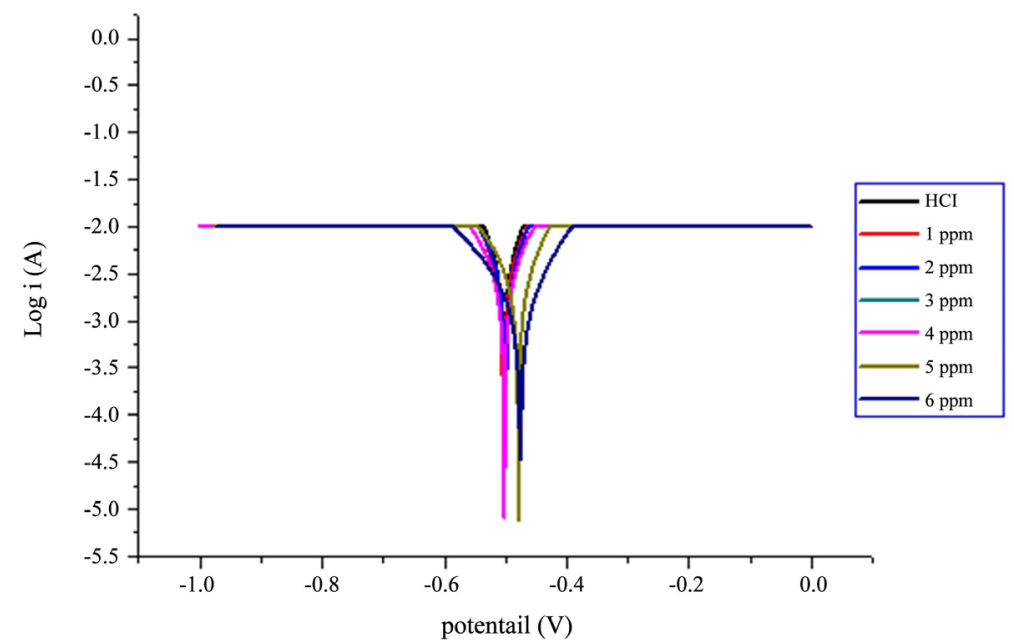

Figure 7. Tafel Plot of GO concentration (1 - $6 \mathrm{ppm}$ ) on C-steel alloy in $318 \mathrm{k}$. 


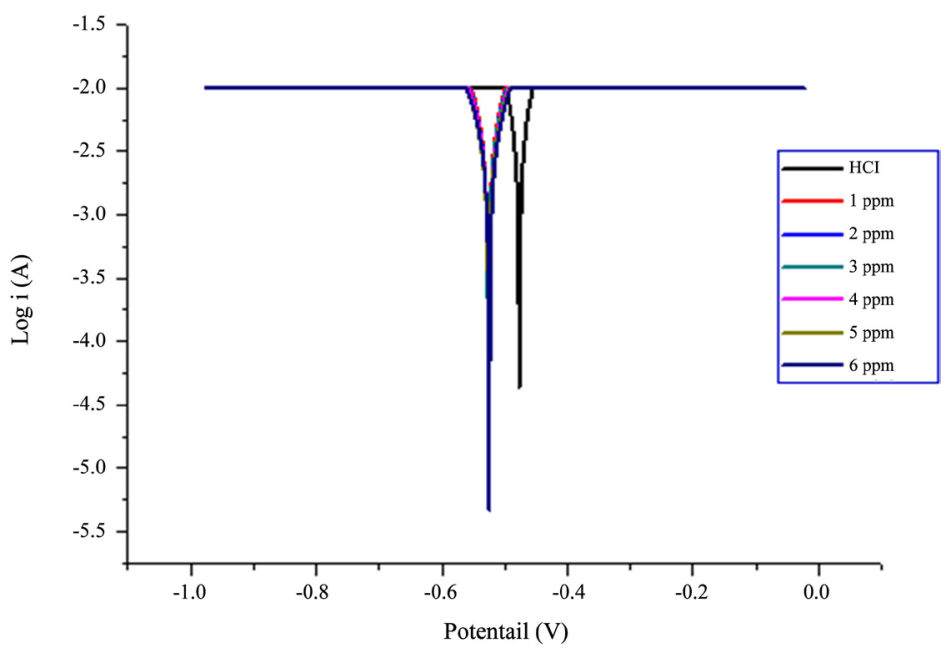

Figure 8. Tafel Plot of GO concentration (1 - $6 \mathrm{ppm})$ on C-steel alloy in $328 \mathrm{k}$.

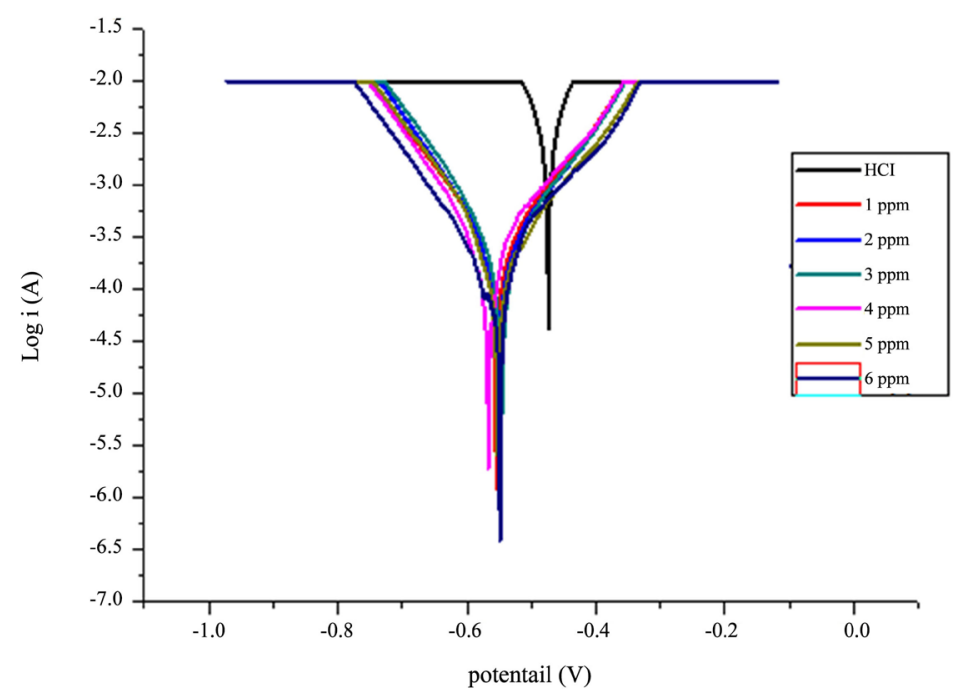

Figure 9. Tafel Plot of GON concentration (1 - 6 ppm) on C-steel alloy in 298 k.

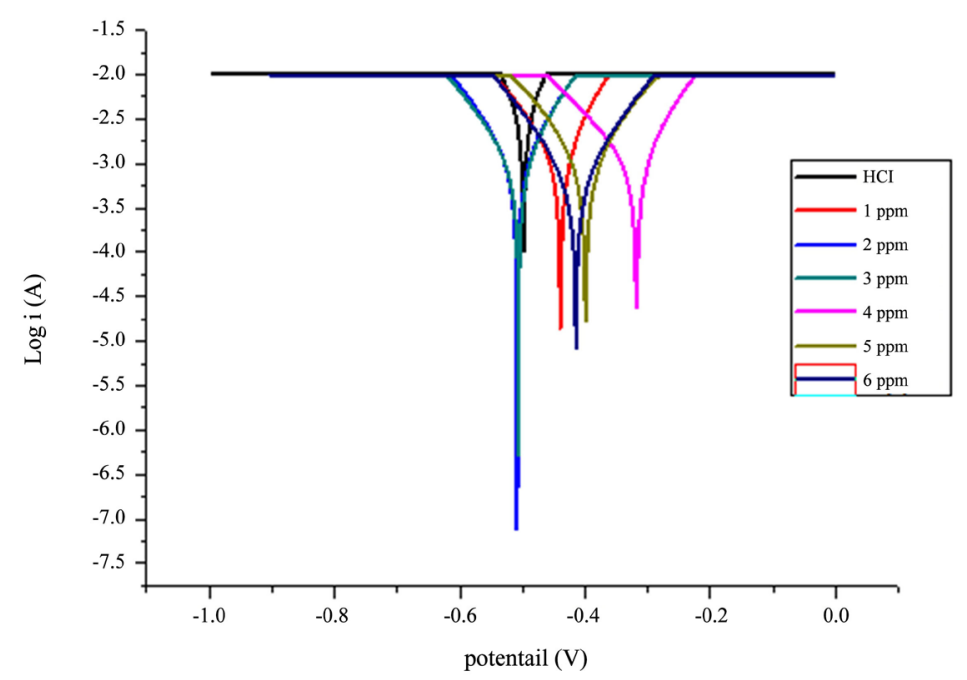

Figure 10. Tafel Plot of GON concentration (1 - $6 \mathrm{ppm}$ ) on C-steel alloy in $308 \mathrm{k}$. 


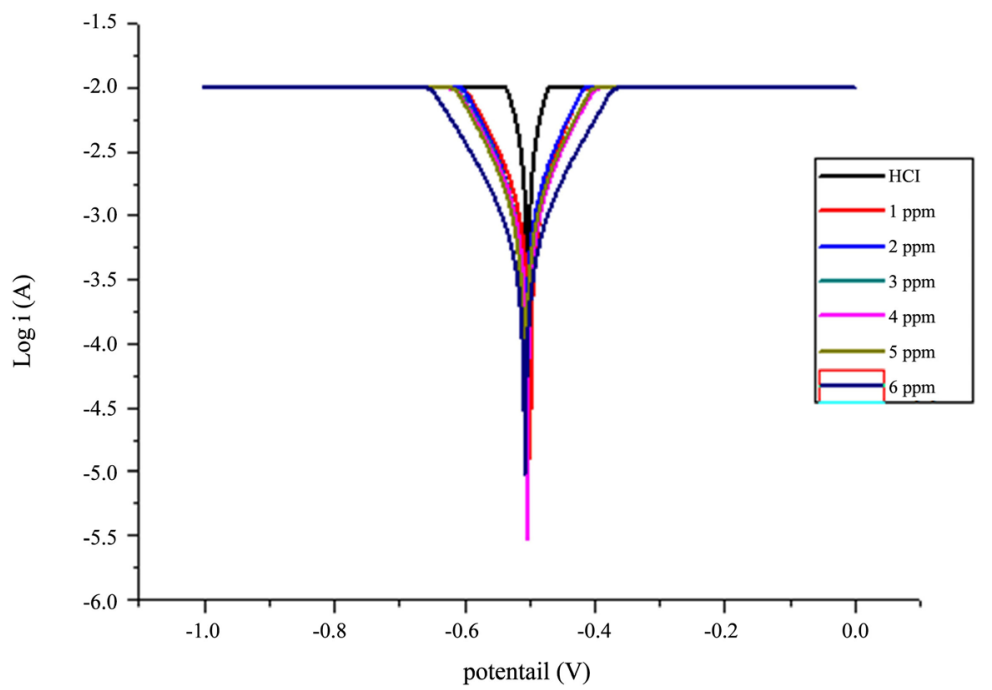

Figure 11. Tafel Plot of GON concentration (1 - $6 \mathrm{ppm})$ on C-steel alloy in $318 \mathrm{k}$.

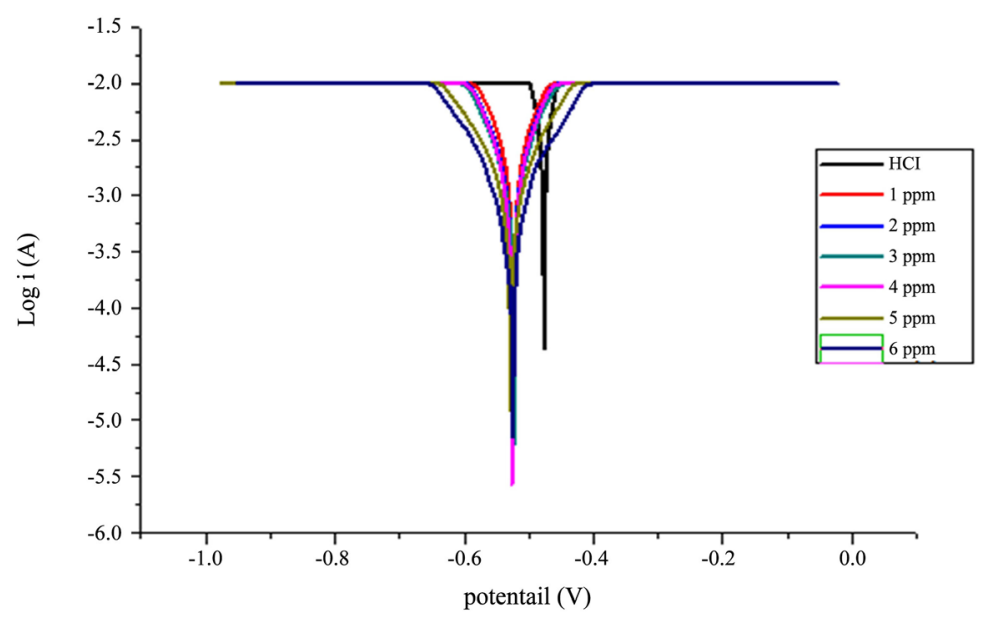

Figure 12. Tafel Plot of GON concentration (1 - 6 ppm) on C-steel alloy in $328 \mathrm{k}$.

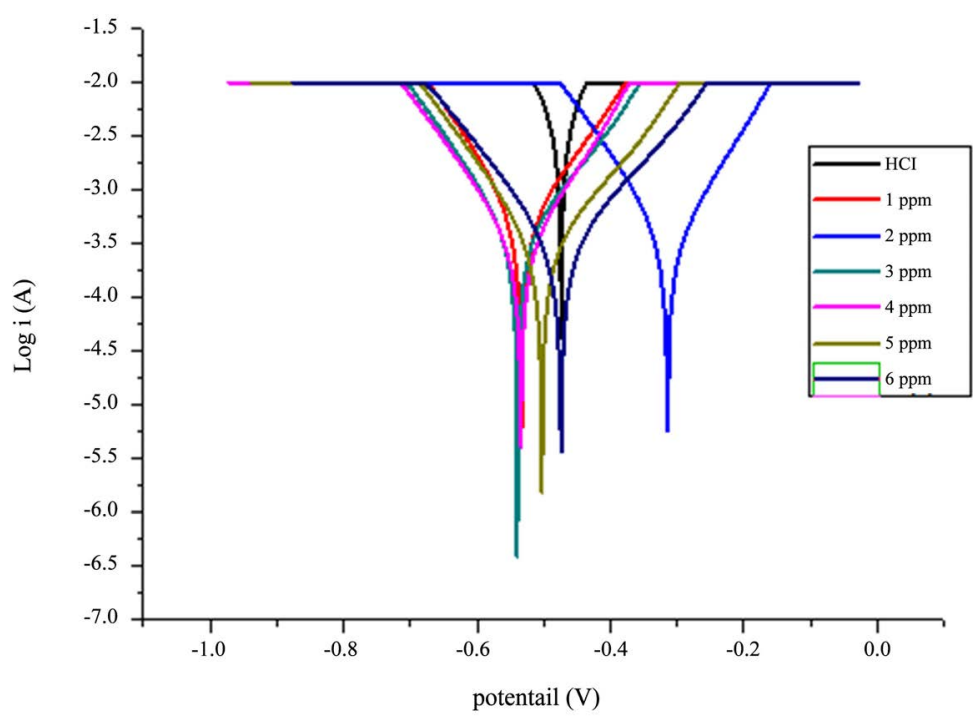

Figure 13. Tafel Plot of GOS concentration (1 - 6 ppm) on C-steel alloy in $298 \mathrm{k}$. 


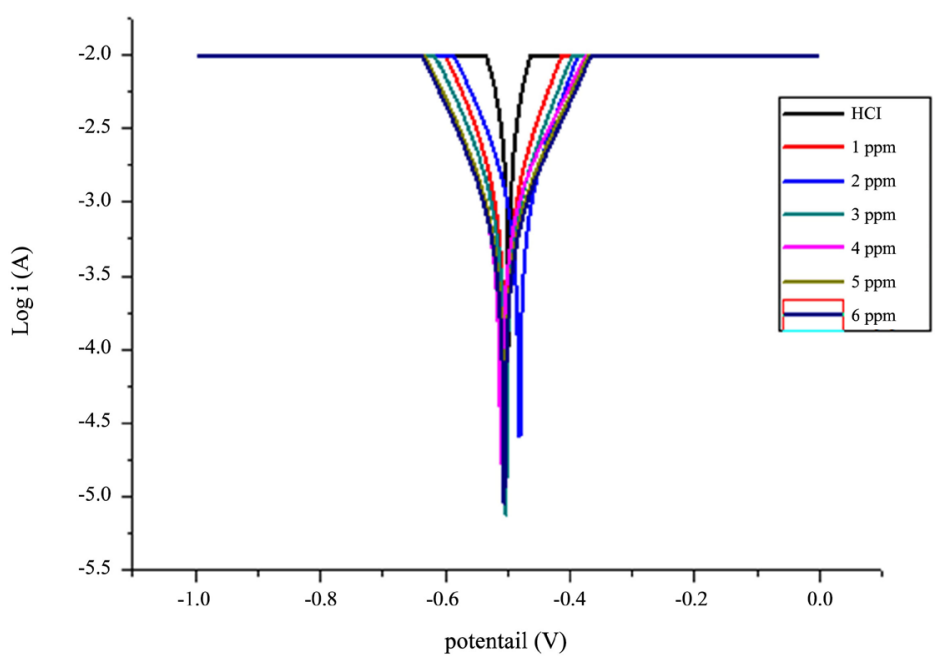

Figure 14. Tafel Plot of GOS concentration (1 - $6 \mathrm{ppm}$ ) on C-steel alloy in $308 \mathrm{k}$.

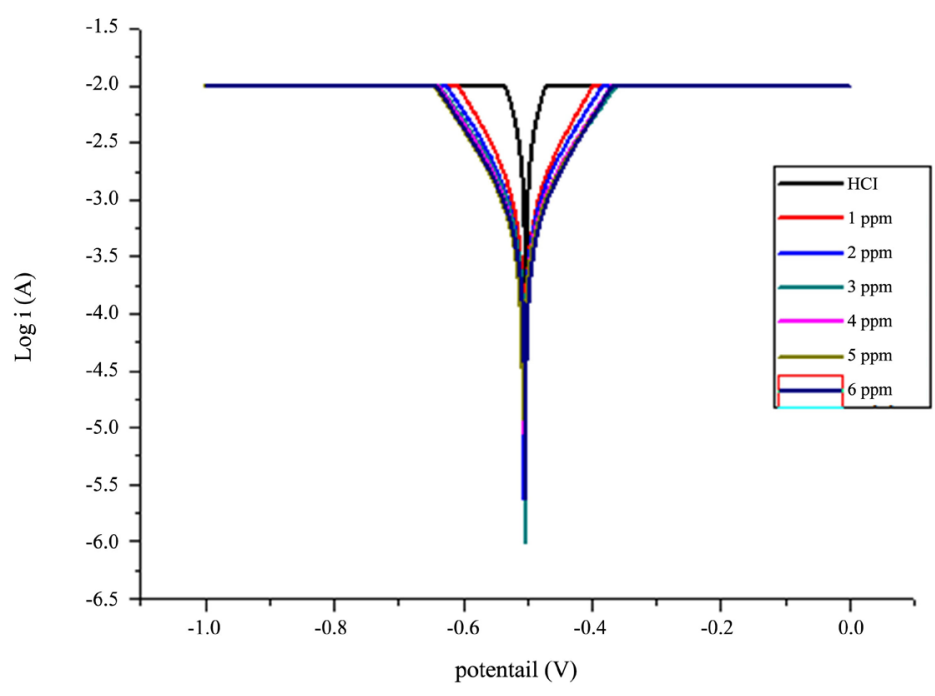

Figure 15. Tafel Plot of GOS concentration (1 - $6 \mathrm{ppm})$ on C-steel alloy in $318 \mathrm{k}$.

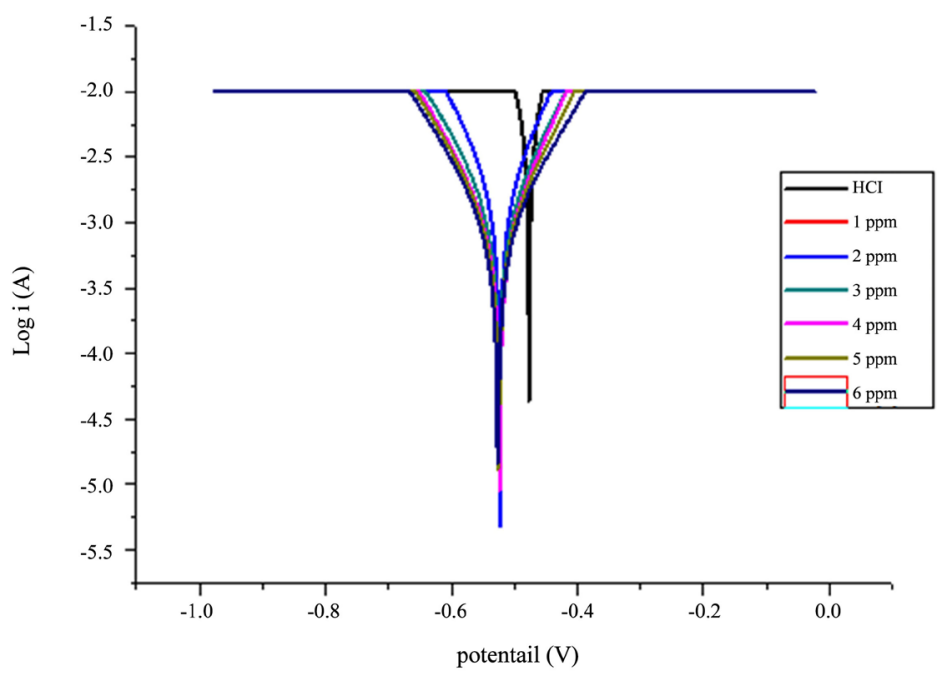

Figure 16. Tafel Plot of GOS concentration (1 - $6 \mathrm{ppm})$ on C-steel alloy in $328 \mathrm{k}$. 
H. H. Radey et al.

Table 1. Tafel parameters for C-steel $1 \mathrm{M} \mathrm{HCl}$ in the absence and presence of different concentrations of GO at different Temp.

\begin{tabular}{|c|c|c|c|c|c|c|c|c|}
\hline Comp. & Temp(K) & $\begin{array}{c}\mathrm{I}_{\text {corr }} \\
\left(\mu \mathrm{A} \cdot \mathrm{Cm}^{-2}\right)\end{array}$ & $\begin{array}{c}\mathrm{CR} \\
\text { (mpy) }\end{array}$ & $\begin{array}{l}\mathrm{R}_{\mathrm{ct}} \\
(\Omega)\end{array}$ & $\begin{array}{l}E_{\text {corr }} \\
(\mathrm{mV})\end{array}$ & $\begin{array}{c}\beta \mathrm{a} \\
(\mathrm{mV} / \mathrm{de} .)\end{array}$ & $\begin{array}{c}-\beta c \\
(\mathrm{mV} / \text { de. })\end{array}$ & Eff.\% \\
\hline $\mathrm{HCl}$ & & 726.98 & 337.12 & 41.82 & -474 & 16.47 & -8.233 & - \\
\hline $1 \mathrm{ppm}$ & & 318.22 & 147.56 & 95.54 & -511 & 12.00 & -7.360 & 56.22 \\
\hline $2 \mathrm{ppm}$ & & 287.22 & 133.2 & 105.9 & -514 & 4.887 & -7.428 & 60.48 \\
\hline $3 \mathrm{ppm}$ & 298 & 234.20 & 108.60 & 129.8 & -510 & 8.046 & -7.212 & 67.78 \\
\hline $4 \mathrm{ppm}$ & & 195.86 & 90.84 & 155.2 & -502 & 8.668 & -7.422 & 73.05 \\
\hline $5 \mathrm{ppm}$ & & 153.25 & 71.04 & 198.4 & -474 & 9.502 & -6.992 & 78.92 \\
\hline $6 \mathrm{ppm}$ & & 138.70 & 64.32 & 219.2 & -494 & 11.45 & -7.621 & 80.92 \\
\hline $\mathrm{HCl}$ & & 801.07 & 371.44 & 37.96 & -498 & 9.312 & -8.233 & - \\
\hline $1 \mathrm{ppm}$ & & 692.07 & 252.36 & 43.94 & -496 & 5.460 & -5.183 & 32.05 \\
\hline $2 \mathrm{ppm}$ & & 544.26 & 245.44 & 55.87 & -507 & 4.321 & -9.522 & 33.92 \\
\hline $3 \mathrm{ppm}$ & 308 & 529.35 & 239.96 & 58.75 & -503 & 8.551 & -1.647 & 35.39 \\
\hline $4 \mathrm{ppm}$ & & 517.51 & 217.16 & 57.45 & -504 & 4.059 & -4.335 & 41.53 \\
\hline $5 \mathrm{ppm}$ & & 467.22 & 211.28 & 64.93 & -505 & 10.86 & -8.097 & 43.11 \\
\hline $6 \mathrm{ppm}$ & & 455.74 & 205.6 & 66.93 & -505 & 3.352 & -1.647 & 44.64 \\
\hline $\mathrm{HCl}$ & & 927.81 & 430.4 & 32.77 & -504 & 4.117 & -1.647 & - \\
\hline $1 \mathrm{ppm}$ & & 719.17 & 333.48 & 42.28 & -506 & 12.34 & -1.647 & 22.51 \\
\hline $2 \mathrm{ppm}$ & & 700.71 & 324.88 & 43.40 & -499 & 8.233 & -1.647 & 49.03 \\
\hline $3 \mathrm{ppm}$ & 318 & 658.22 & 305.2 & 46.20 & -506 & 4.231 & -1.647 & 29.08 \\
\hline $4 \mathrm{ppm}$ & & 516.45 & 239.48 & 58.55 & -506 & 4.219 & -1.647 & 44.35 \\
\hline $5 \mathrm{ppm}$ & & 442.13 & 205 & 68.77 & -491 & 8.233 & -7.207 & 52.36 \\
\hline $6 \mathrm{ppm}$ & & 210.06 & 97.4 & 74.48 & -490 & 8.851 & -7.464 & 77.36 \\
\hline $\mathrm{HCl}$ & & 1396.45 & 647.6 & 21.77 & -477 & 16.47 & -8.233 & - \\
\hline $1 \mathrm{ppm}$ & & 1115.03 & 517.2 & 27.27 & -523 & 16.47 & -1.647 & 20.13 \\
\hline $2 \mathrm{ppm}$ & & 1032.43 & 479.6 & 29.39 & -522 & 8.233 & -1.647 & 25.94 \\
\hline $3 \mathrm{ppm}$ & 328 & 1008.99 & 446.8 & 30.13 & -524 & 8.233 & -1.647 & 31.00 \\
\hline $4 \mathrm{pmm}$ & & 953.14 & 442 & 31.90 & -515 & 8.233 & -1.647 & 31.74 \\
\hline $5 \mathrm{ppm}$ & & 871.48 & 400.32 & 36.98 & -503 & 8.233 & -1.647 & 38.18 \\
\hline $6 \mathrm{ppm}$ & & 840.95 & 389.92 & 39.16 & -525 & 8.233 & -1.647 & 39.79 \\
\hline
\end{tabular}

Table 2. Tafel parameters for C-steel $1 \mathrm{M} \mathrm{HCl}$ in the absence and presence of different concentrations of GON at different Temp.

\begin{tabular}{ccccccccc}
\hline Comp. & Temp $(\mathrm{K})$ & $\begin{array}{c}\mathrm{I}_{\mathrm{corr}} \\
\left(\mu \mathrm{A} \cdot \mathrm{Cm}^{-2}\right)\end{array}$ & $\begin{array}{c}\mathrm{CR} \\
(\mathrm{mpy})\end{array}$ & $\begin{array}{c}\mathrm{R}_{\mathrm{ct}} \\
(\Omega)\end{array}$ & $\begin{array}{c}\mathrm{E}_{\text {corr }} \\
(\mathrm{mV})\end{array}$ & $\begin{array}{c}\beta \mathrm{a} \\
(\mathrm{mV} / \mathrm{de} .)\end{array}$ & $\begin{array}{c}-\beta \mathrm{c} \\
(\mathrm{mV} / \mathrm{de} .)\end{array}$ & $\begin{array}{c}\mathrm{Eff} \% \\
\mathrm{HCl}\end{array}$ \\
& & 726.98 & 337.12 & 41.82 & -474 & 16.47 & -8.233 & - \\
$1 \mathrm{ppm}$ & 298 & 32.97 & 15.28 & 92.23 & -490 & 7.885 & -8.680 & 95.46 \\
$2 \mathrm{ppm}$ & & 29.54 & 13.70 & 102.9 & -496 & 7.449 & -9.793 & 95.93 \\
$3 \mathrm{ppm}$ & & 28.84 & 13.37 & 105.4 & -481 & 8.276 & -11.018 & 96.03 \\
\hline
\end{tabular}




\section{Continued}

\begin{tabular}{|c|c|c|c|c|c|c|c|c|}
\hline $4 \mathrm{ppm}$ & & 27.23 & 12.58 & 111.7 & -499 & 7.027 & -9.075 & 96.26 \\
\hline $5 \mathrm{ppm}$ & & 25.50 & 11.82 & 119.2 & -488 & 8.807 & -9.314 & 96.49 \\
\hline $6 \mathrm{ppm}$ & & 22.11 & 10.22 & 137.9 & -482 & 6.658 & -9.721 & 96.96 \\
\hline $\mathrm{HCl}$ & & 801.07 & 371.44 & 37.96 & -498 & 9.312 & -8.233 & - \\
\hline $1 \mathrm{ppm}$ & & 188.76 & 87.52 & 161.1 & -459 & 6.660 & -9.039 & 76.43 \\
\hline $2 \mathrm{ppm}$ & & 153.49 & 71.20 & 198.0 & -532 & 10.57 & -9.814 & 80.83 \\
\hline $3 \mathrm{ppm}$ & 308 & 148.76 & 68.96 & 204.5 & -524 & 10.76 & -9.371 & 81.43 \\
\hline $4 \mathrm{ppm}$ & & 142.72 & 66.20 & 213.0 & -414 & 10.13 & -7.539 & 82.17 \\
\hline $5 \mathrm{ppm}$ & & 128.52 & 59.60 & 236.6 & -448 & 9.286 & -8.969 & 83.95 \\
\hline $6 \mathrm{ppm}$ & & 84.09 & 38.992 & 361.6 & -418 & 10.24 & -9.676 & 89.50 \\
\hline $\mathrm{HCl}$ & & 927.81 & 430.4 & 32.77 & -504 & 4.117 & -1.647 & - \\
\hline $1 \mathrm{ppm}$ & & 188.64 & 87.48 & 162.1 & -520 & 8.209 & -9.489 & 79.67 \\
\hline $2 \mathrm{ppm}$ & & 187.22 & 86.80 & 162.4 & -530 & 10.14 & -9.701 & 79.83 \\
\hline $3 \mathrm{ppm}$ & 318 & 161.78 & 75.48 & 186.8 & -526 & 9.312 & -9.078 & 82.46 \\
\hline $4 \mathrm{ppm}$ & & 160.12 & 74.24 & 189.9 & -522 & 9.431 & -9.059 & 82.75 \\
\hline $5 \mathrm{ppm}$ & & 150.41 & 69.76 & 202.1 & -528 & 9.369 & -9.610 & 83.79 \\
\hline $6 \mathrm{ppm}$ & & 81.62 & 37.84 & 372.5 & -516 & 9.473 & -9.171 & 91.20 \\
\hline $\mathrm{HCl}$ & & 1396.45 & 647.6 & 21.77 & -477 & 16.47 & -8.233 & - \\
\hline $1 \mathrm{ppm}$ & & 451.24 & 209.28 & 67.3 & -500 & 2.720 & -1.727 & 67.68 \\
\hline $2 \mathrm{ppm}$ & & 352.07 & 163.24 & 86.8 & -507 & 5.448 & -1.563 & 74.79 \\
\hline 3 ppm & 328 & 312.90 & 144.84 & 97.1 & -501 & 2.204 & -4.590 & 77.63 \\
\hline $4 \mathrm{ppm}$ & & 266.04 & 121.36 & 114.3 & -503 & 8.827 & -2.043 & 81.26 \\
\hline $5 \mathrm{pmm}$ & & 189.70 & 89.48 & 157.6 & -492 & 9.529 & -8.213 & 86.18 \\
\hline $6 \mathrm{ppm}$ & & 113.15 & 52.48 & 268.7 & -493 & 9.509 & -8.415 & 91.89 \\
\hline
\end{tabular}

Table 3. Tafel parameters for C-steel $1 \mathrm{M} \mathrm{HCl}$ in the absence and presence of different concentrations of GOS at different Temp.

\begin{tabular}{|c|c|c|c|c|c|c|c|c|}
\hline Comp. & Temp (K) & $\begin{array}{c}\mathrm{I}_{\text {corr }} \\
\left(\mu \mathrm{A} \cdot \mathrm{Cm}^{-2}\right)\end{array}$ & $\begin{array}{c}\text { CR } \\
\text { (mpy) }\end{array}$ & $\begin{array}{l}\mathrm{R}_{\mathrm{ct}} \\
(\Omega)\end{array}$ & $\begin{array}{l}E_{\text {corr }} \\
(\mathrm{mV})\end{array}$ & $\begin{array}{c}\beta \mathrm{a} \\
(\mathrm{mV} / \text { de. })\end{array}$ & $\begin{array}{c}-\beta c \\
(\mathrm{mV} / \text { de. })\end{array}$ & Eff.\% \\
\hline $\mathrm{HCl}$ & & 726.98 & 337.12 & 41.82 & -474 & 16.47 & -8.233 & - \\
\hline $1 \mathrm{ppm}$ & & 65.07 & 30.168 & 54.96 & -509 & 8.586 & -1.003 & 91.05 \\
\hline $2 \mathrm{ppm}$ & & 45.89 & 21.28 & 66.25 & -291 & 10.24 & -9.752 & 93.68 \\
\hline $3 \mathrm{ppm}$ & 298 & 43.31 & 20.11 & 70.19 & -518 & 7.881 & -9.763 & 94.03 \\
\hline $4 \mathrm{ppm}$ & & 37.83 & 17.54 & 80.38 & -508 & 9.645 & -9.297 & 94.79 \\
\hline $5 \mathrm{ppm}$ & & 31.85 & 14.76 & 95.49 & -520 & 7.507 & -9.526 & 95.62 \\
\hline $6 \mathrm{ppm}$ & & 27.05 & 14.18 & 99.37 & -522 & 7.444 & -8.909 & 95.79 \\
\hline $\mathrm{HCl}$ & 308 & 801.07 & 371.44 & 37.96 & -498 & 9.312 & -8.233 & - \\
\hline $1 \mathrm{ppm}$ & & 168.17 & 77.96 & 180.8 & -510 & 11.12 & -9.724 & 79.01 \\
\hline
\end{tabular}




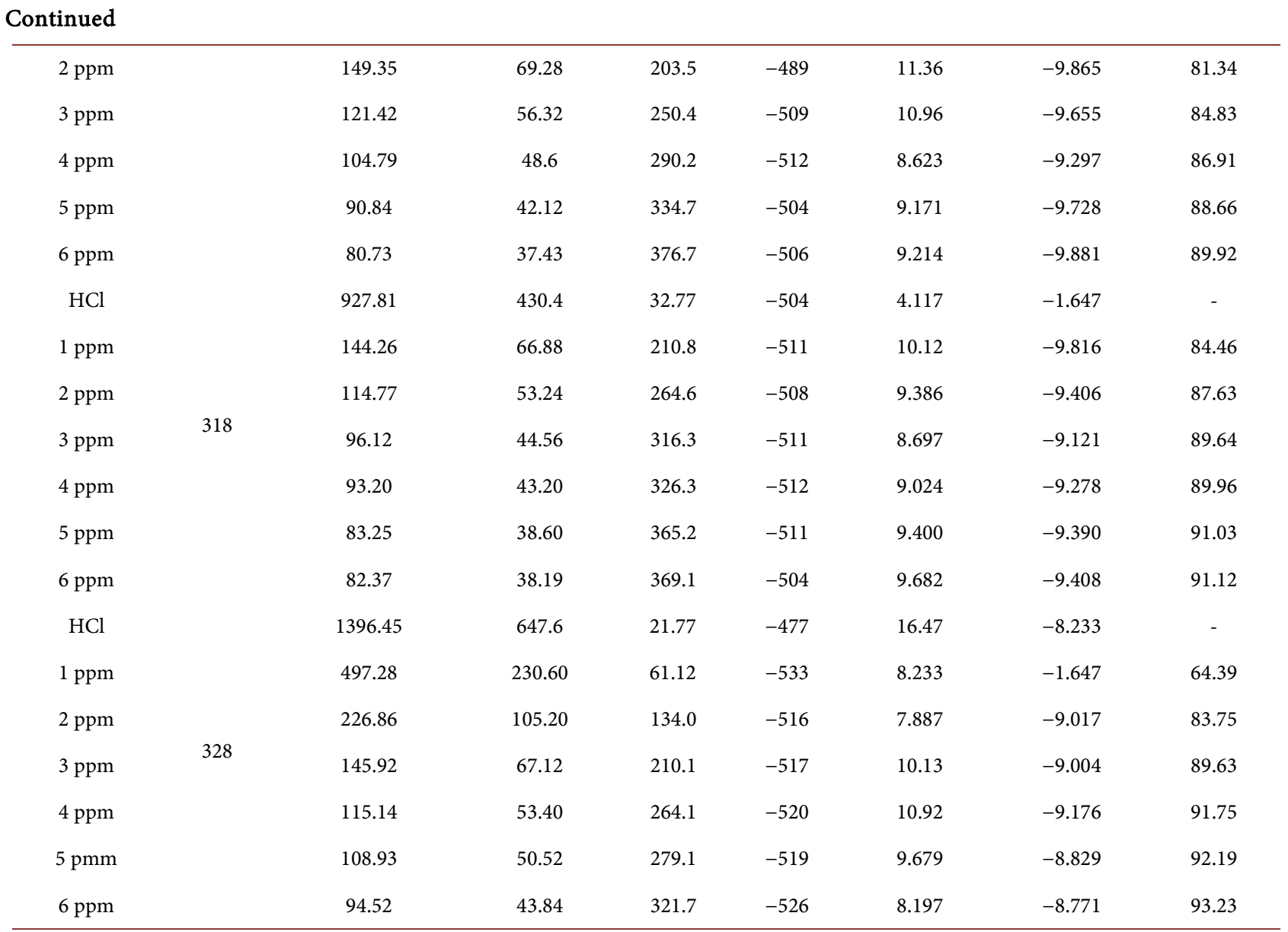

It is evident from Tables 1-3 from $\mathrm{E}_{\text {corr }}$ at constant temperature compared with $\mathrm{E}_{\text {corr }}$ to blank at $298 \mathrm{~K}$ for graphene oxide (GO) the $\mathrm{E}_{\text {corr }}$ decrease at all concentration compared with $\mathrm{E}_{\text {corr }}$ to blank that value $(-474 \mathrm{mv}$ ) but this decreasing less to $(89 \mathrm{mv})$ so inhibitor behavior it mix inhibitor, as observed from previous studies [22] [23] [24], if the difference $\mathrm{E}_{\text {corr }}$ between blank and inhibitor ( $89 \mathrm{mv}$ ) decreasing less the inhibitor is mix, also $E_{\text {corr }}$ other organic inhibitors GON, GOSdecreasing less ( $89 \mathrm{mv}$ ) compared with $\mathrm{E}_{\text {corr }}$ blank that mean It behavesmix inhibitor at all concentration ( $1-6 \mathrm{ppm})$ and temperature (298 - $328 \mathrm{~K})$. In this study used very small concentration organic inhibitors it gives high efficiency inhibitor, show graphene oxide have less efficiency inhibitor (20.13\%) at (1 ppm) and $(80.92 \%)$ at $(6 \mathrm{ppm})$ in spite of containing carboxylic group in graphene oxide layer GON, GOS have efficiency inhibitor (67.68\%, 64.39\%), respectively at $(1 \mathrm{ppm})$ and $(96.96 \%, 95.79 \%)$ respectively at $(6 \mathrm{ppm})$ at different temperature.

The $R_{c t}$ values of the inhibited are increase as the concentration of the inhibitors increases on the other hand increase in efficiency inhibitors due to productive carbon steel surface to resist polarization, and the anodic reaction the dissolution of Iron in carbon steel alloy to reduce in present inhibitors the signification that increase of Tafel $\left(\beta_{\mathrm{a}}\right)$ [25]. 


\subsubsection{Effect of Temperature}

The study of effect of temperature on the corrosion rate to the organic inhibtors on carbon steel alloylwere immersed in $1 \mathrm{M}$ hydrochloric acid with different concentration (1- $6 \mathrm{ppm}$ ) of inhibitor at temperature ranging from $298 \mathrm{~K}, 308 \mathrm{~K}$, $318 \mathrm{~K}$, and $328 \mathrm{~K}$, the activation energy value was calculated from Arrhenius equation [26]

$$
\ln \mathrm{CR}=\ln \mathrm{A}-\frac{-\mathrm{Ea}}{\mathrm{RT}}
$$

where: $\mathrm{CR}=$ corrosion rate (mpy), $\mathrm{Ea}=$ activation energy $(\mathrm{KJ} / \mathrm{mol})$;

$\mathrm{A}=$ frequency factor, $\mathrm{R}=$ molar gas constant $\left(8.3143 \mathrm{~J} \cdot \mathrm{K}^{-1} \cdot \mathrm{mol}^{-1}\right)$;

$\mathrm{T}=$ temperature $(\mathrm{K})$.

Figure 17, Figure 18, Figure 19 show good relationship between (ln CR) vs $\left(1 / \mathrm{T} \mathrm{K}^{-1}\right)$ for without and with inhibitors organic compound in $1 \mathrm{M} \mathrm{HCl}$ solution, straight lines were obtained with slope of $(-\mathrm{Ea} / \mathrm{R})$, the activation energy was calculated from slope of Arrhenius plot. the activation energy as the concentration of inhibitor increase which indicates physical adsorption [27] and it due to be corrosion reaction in which charge transfer has been blocked by the adsorption of inhibitor molecular on the carbon steel surface. The activation energy (Ea) value in the presence of corrosion inhibitors is higher than in absence of inhibitor also Ea increase with increasing concentration in inhibitors.

Enthalpy $(\Delta \mathrm{H})$ and entropy $(\Delta \mathrm{S})$ of activation have been calculated from the following equation

$$
\mathrm{CR}=\frac{\mathrm{RT}}{\mathrm{Nh}} \operatorname{Ln}\left(\frac{\Delta \mathrm{S}^{*}}{\mathrm{R}}\right) \operatorname{Ln}\left(\frac{-\Delta \mathrm{H}^{*}}{\mathrm{RT}}\right)
$$

$\mathrm{CR}=$ corrosion rate (mpy), $\Delta \mathrm{H}=$ Enthalpy $(\mathrm{KJ} / \mathrm{mol}), \Delta \mathrm{S}=$ entropy $(\mathrm{J} / \mathrm{mol}$, $\mathrm{K}), \mathrm{R}=$ molar gas constant $\left(8.3143 \mathrm{~J} \cdot \mathrm{K}^{-1} \cdot \mathrm{mol}^{-1}\right), \mathrm{T}=$ temperature $(\mathrm{K}), \mathrm{N}=$ Avogadro number $\left(6.022 \times 10^{23} \mathrm{~mol}^{-1}\right) ; \mathrm{h}=$ plank, constant $\left(6.62 \times 10^{-34} \mathrm{~J} \cdot \mathrm{s}\right)$.

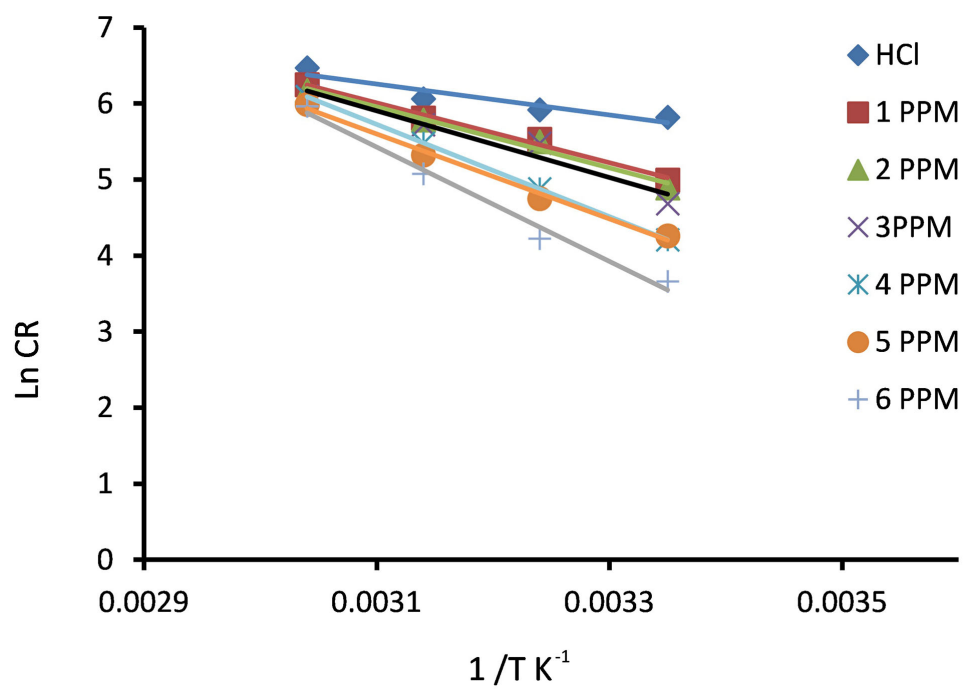

Figure 17. Adsorption isotherm plot for $\ln (\mathrm{CR})$ vs $1 / \mathrm{T}$ to the GO. 


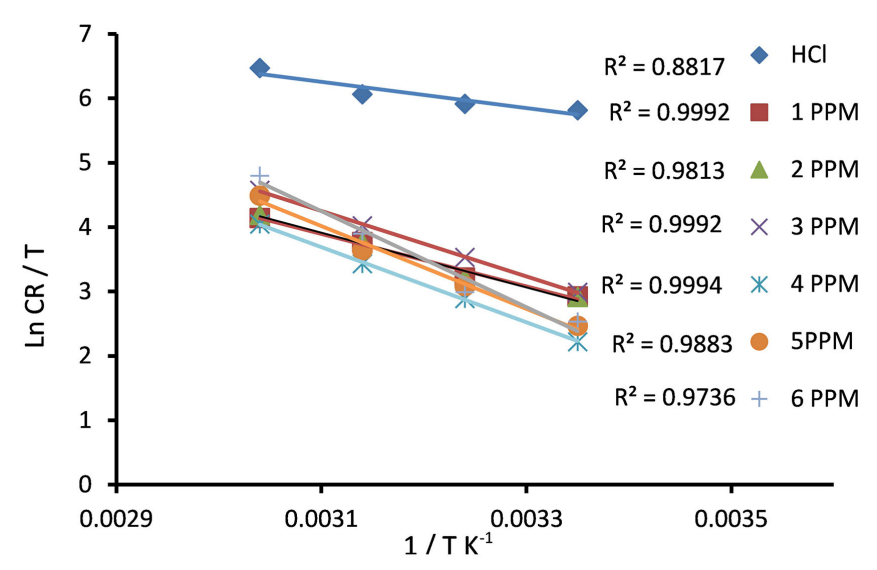

Figure 18. Adsorption isotherm plot for $\ln (\mathrm{CR})$ vs $1 / \mathrm{T}$ GON.

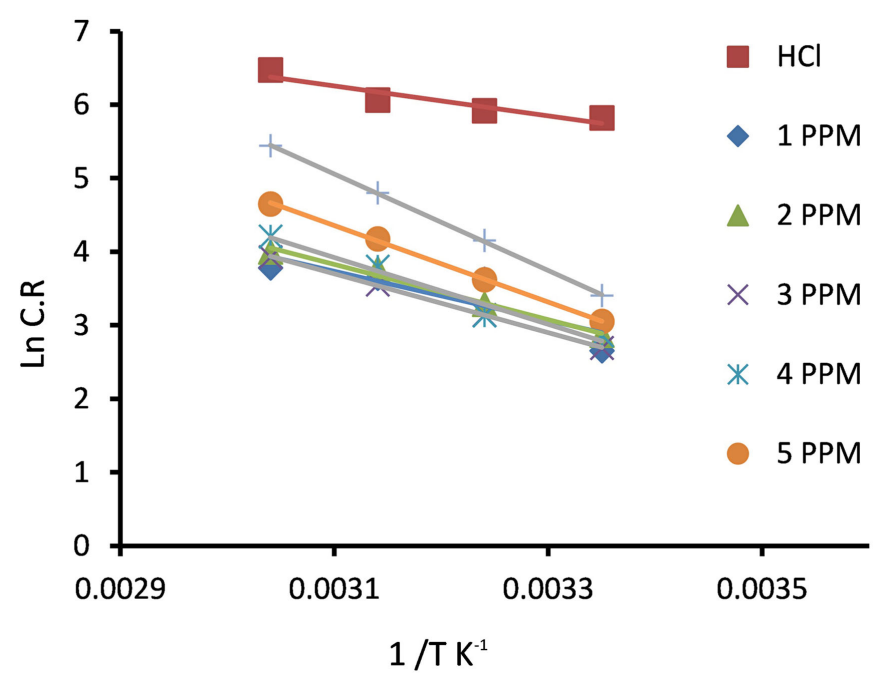

Figure 19. Adsorption isotherm plot for $\ln (\mathrm{CR})$ vs 1/T GOS.

Figure 20, Figure 21, Figure 22 are the plots of $(\ln \mathrm{CR} / \mathrm{T})$ vs $\left(1 / \mathrm{T} \mathrm{K}^{-1}\right)$ for carbon steel in with and without inhibitors organic compound in $1 \mathrm{~N} \mathrm{HCl}$ solution. Curves showed straight lines with slope $\left(\Delta \mathrm{H}^{*} / \mathrm{R}\right)$ and intercept $\left(\Delta \mathrm{S}^{*} / \mathrm{R}\right)$. The positive value of $\Delta \mathrm{H}^{*}$ reflect that the process of desorption of the inhibitors on the surface is an endothermic process (chemical adsorption) and it has been clearly observed that the value of $\Delta \mathrm{H}^{*}$ increasing as the concentration inhibitor increase. The entropy of activation in mentioned table clear that these values increased positively in the presence of inhibitor than in its absence. The increase of reveals that an increase in disordering takes place from reactant to the activated complex [28] (Tables 4-6).

\subsubsection{Adsorption Isotherm}

The adsorption isotherms provide basic information about the interaction between the inhibitor and the Carbon steel surface. In this study fit with Langmuir isotherm and the Langmuir isotherm best model to gives of value of $\left(\mathrm{R}^{2}\right)$ correlation coefficient equal one or approaching one, through $\mathrm{R}^{2}$ to found Langmuir 
isotherm was calculated using the equation below [29]

$$
\frac{\theta}{1-\theta}=K . C
$$

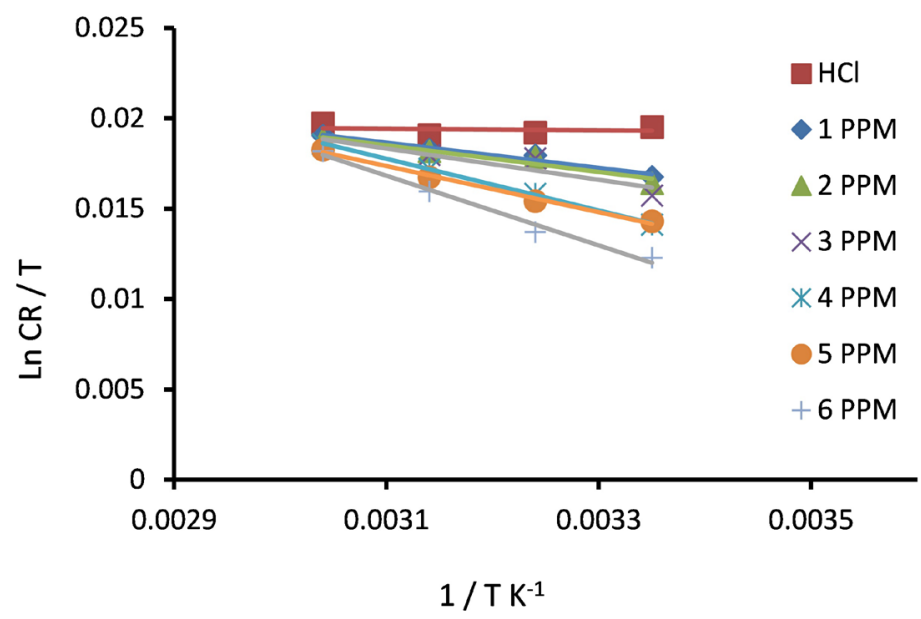

Figure 20. Adsorption isotherm plot for $(\ln \mathrm{CR} / \mathrm{T})$ vs $1 / \mathrm{T}$ to the GO.

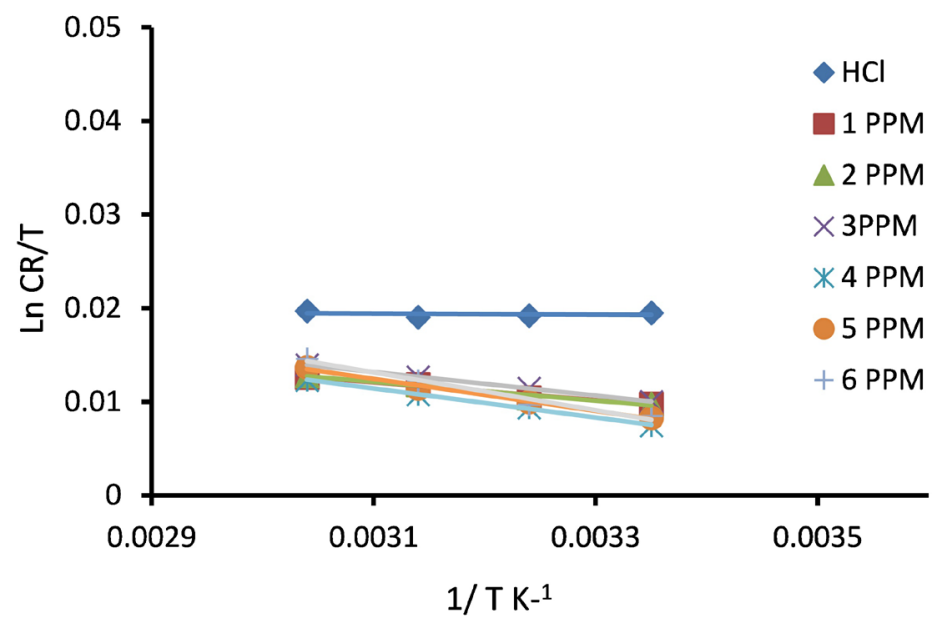

Figure 21. Adsorption isotherm plot for (ln CR/T) vs 1/T GON.

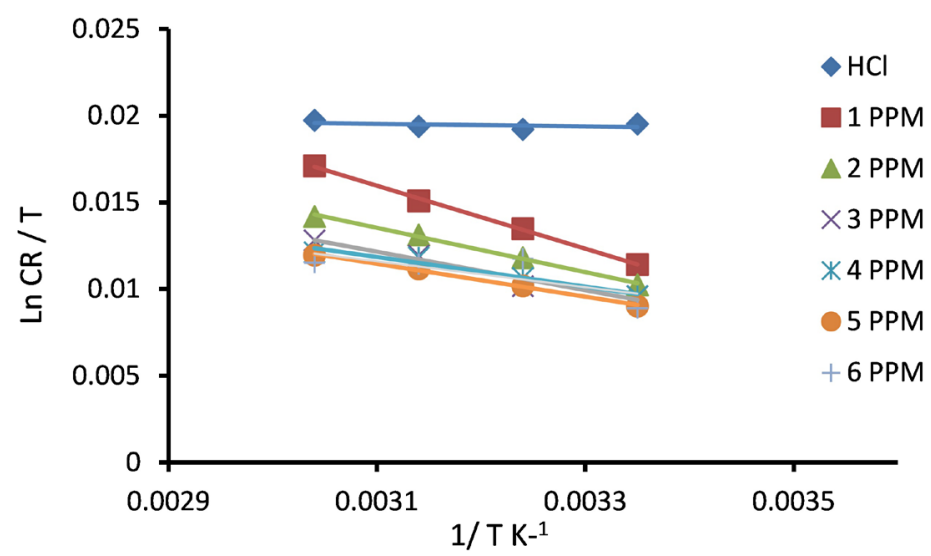

Figure 22. Adsorption isotherm plot for $(\ln \mathrm{CR} / \mathrm{T})$ vs $1 / \mathrm{T}$ GOS. 
Table 4. Kinetic parameters $\mathrm{Ea}^{*}, \Delta \mathrm{H}^{\star}, \Delta \mathrm{G}^{\star}$ and $\Delta \mathrm{S}^{\star}$ for carbon steel of GO in $1 \mathrm{M} \mathrm{HCl}$ at $(1,6 \mathrm{ppm})$.

\begin{tabular}{|c|c|c|c|c|c|c|c|}
\hline \multirow{2}{*}{$\begin{array}{l}\text { Conc. } \\
\text { (ppm) }\end{array}$} & \multirow{2}{*}{$\mathrm{Ea}^{*}(\mathrm{KJ} / \mathrm{mol})$} & \multirow{2}{*}{$\begin{array}{c}\Delta \mathrm{H}^{\star} \\
(\mathrm{KJ} / \mathrm{mol})\end{array}$} & \multirow{2}{*}{$\Delta \mathrm{s}^{*}(\mathrm{~J} / \mathrm{mol} \cdot \mathrm{K})$} & \multicolumn{4}{|c|}{$\mathrm{G}^{*}(\mathrm{KJ} / \mathrm{mol} \cdot \mathrm{K}) \Delta$} \\
\hline & & & & $298 \mathrm{~K}$ & $308 \mathrm{~K}$ & $318 \mathrm{~K}$ & $328 \mathrm{~K}$ \\
\hline Blank & 17 & 3.46 & 17.23 & -1.67 & -1.84 & -2.01 & -2.19 \\
\hline $1 \mathrm{ppm}$ & 33 & 58.23 & 33.56 & 48.23 & 47.89 & 47.56 & 47.22 \\
\hline $2 \mathrm{ppm}$ & 33 & 61.21 & 34.36 & 50.97 & 50.62 & 50.28 & 49.94 \\
\hline $3 \mathrm{ppm}$ & 36 & 71.88 & 37.52 & 60.70 & 60.32 & 59.95 & 59.57 \\
\hline $4 \mathrm{ppm}$ & 50 & 118.94 & 51.64 & 103.55 & 103.03 & 102.52 & 102.00 \\
\hline $5 \mathrm{ppm}$ & 57 & 140.93 & 57.97 & 123.66 & 123.08 & 122.50 & 121.92 \\
\hline $6 \mathrm{ppm}$ & 62 & 160.41 & 63.72 & 141.42 & 140.79 & 140.15 & 139.51 \\
\hline
\end{tabular}

Table 5. Kinetic parameters $\mathrm{Ea}^{*}, \Delta \mathrm{H}^{*}, \Delta \mathrm{G}^{*}$ and $\Delta \mathrm{S}^{\star}$ for carbon steel of GON in $1 \mathrm{M} \mathrm{HCl}$ at $(1,6 \mathrm{ppm})$.

\begin{tabular}{|c|c|c|c|c|c|c|c|}
\hline \multirow{2}{*}{$\begin{array}{l}\text { Conc. } \\
\text { (ppm) }\end{array}$} & \multirow{2}{*}{$\mathrm{Ea}^{*}(\mathrm{KJ} / \mathrm{mol})$} & \multirow{2}{*}{$\begin{array}{c}\Delta \mathrm{H}^{*} \\
(\mathrm{KJ} / \mathrm{mol})\end{array}$} & \multirow{2}{*}{$\Delta \mathrm{s}^{*}(\mathrm{~J} / \mathrm{mol} \cdot \mathrm{K})$} & \multicolumn{4}{|c|}{$\mathrm{G}^{*}(\mathrm{KJ} / \mathrm{mol} \cdot \mathrm{K}) \Delta$} \\
\hline & & & & $298 \mathrm{~K}$ & $308 \mathrm{~K}$ & $318 \mathrm{~K}$ & $328 \mathrm{~K}$ \\
\hline Blank & 16.88 & 3.46 & 17.23 & -1.67 & -1.84 & -2.01 & -2.19 \\
\hline $1 \mathrm{ppm}$ & 33.81 & 79.15 & 34.58 & 68.85 & 68.50 & 68.16 & 67.81 \\
\hline $2 \mathrm{ppm}$ & 35.34 & 83.90 & 36.10 & 73.15 & 72.78 & 72.42 & 72.06 \\
\hline $3 \mathrm{ppm}$ & 42.26 & 104.14 & 43.25 & 91.25 & 90.82 & 90.39 & 89.96 \\
\hline $4 \mathrm{ppm}$ & 48.58 & 129.71 & 49.73 & 114.89 & 114.40 & 113.90 & 113.400 \\
\hline $5 \mathrm{ppm}$ & 53.41 & 142.45 & 54.51 & 126.20 & 125.66 & 125.11 & 124.57 \\
\hline $6 \mathrm{ppm}$ & 61.92 & 168.26 & 63.10 & 149.46 & 148.83 & 148.20 & 147.57 \\
\hline
\end{tabular}

Table 6. Kinetic parameters $\mathrm{Ea}^{\star}, \Delta \mathrm{H}^{\star}, \Delta \mathrm{G}^{*}$ and $\Delta \mathrm{S}^{\star}$ for carbon steel of GOS in $1 \mathrm{M} \mathrm{HCl}$ at $(1,6 \mathrm{ppm})$.

\begin{tabular}{|c|c|c|c|c|c|c|c|}
\hline \multirow{2}{*}{$\begin{array}{l}\text { Conc. } \\
\text { (ppm) }\end{array}$} & \multirow{2}{*}{$\mathrm{Ea}^{*}(\mathrm{KJ} / \mathrm{mol})$} & \multirow{2}{*}{$\begin{array}{c}\Delta \mathrm{H}^{*} \\
(\mathrm{KJ} / \mathrm{mol})\end{array}$} & \multirow{2}{*}{$\Delta \mathrm{s}^{*}(\mathrm{~J} / \mathrm{mol} \cdot \mathrm{K})$} & \multicolumn{4}{|c|}{$\mathrm{G}^{*}(\mathrm{KJ} / \mathrm{mol} \cdot \mathrm{K}) \Delta$} \\
\hline & & & & $298 \mathrm{~K}$ & $308 \mathrm{~K}$ & $318 \mathrm{~K}$ & $328 \mathrm{~K}$ \\
\hline Blank & 16.88 & 3.46 & 17.23 & -1.67 & -1.84 & -2.01 & -2.19 \\
\hline $1 \mathrm{ppm}$ & 27.75 & 70.66 & 31.71 & 61.21 & 60.90 & 60.58 & 60.26 \\
\hline $2 \mathrm{ppm}$ & 31.20 & 71.53 & 32.05 & 61.98 & 61.66 & 61.34 & 61.02 \\
\hline $3 \mathrm{ppm}$ & 33.28 & 79.26 & 34.11 & 69.09 & 68.75 & 68.41 & 68.07 \\
\hline $4 \mathrm{ppm}$ & 37.75 & 91.77 & 38.56 & 80.28 & 79.90 & 79.51 & 79.12 \\
\hline $5 \mathrm{ppm}$ & 43.22 & 106.61 & 44.28 & 93.41 & 92.97 & 92.53 & 92.08 \\
\hline $6 \mathrm{ppm}$ & 54.63 & 138.44 & 55.96 & 121.77 & 121.21 & 120.65 & 120.09 \\
\hline
\end{tabular}

where surface coverage $(\theta)$ for various inhibitor concentrations, $\mathrm{K}_{a d s}$ the adsorptive equilibrium constant, $\mathrm{C}$ is the concentration of the inhibitor, the isotherms at different temperatures for different concentration of organic compound in $1 \mathrm{M} \mathrm{HCl}$, show date in Tables 7-9 and Figures 23-25.

The equilibrium constant for the adsorption process was related to the standardfree energy of adsorption by the expression [30]. 


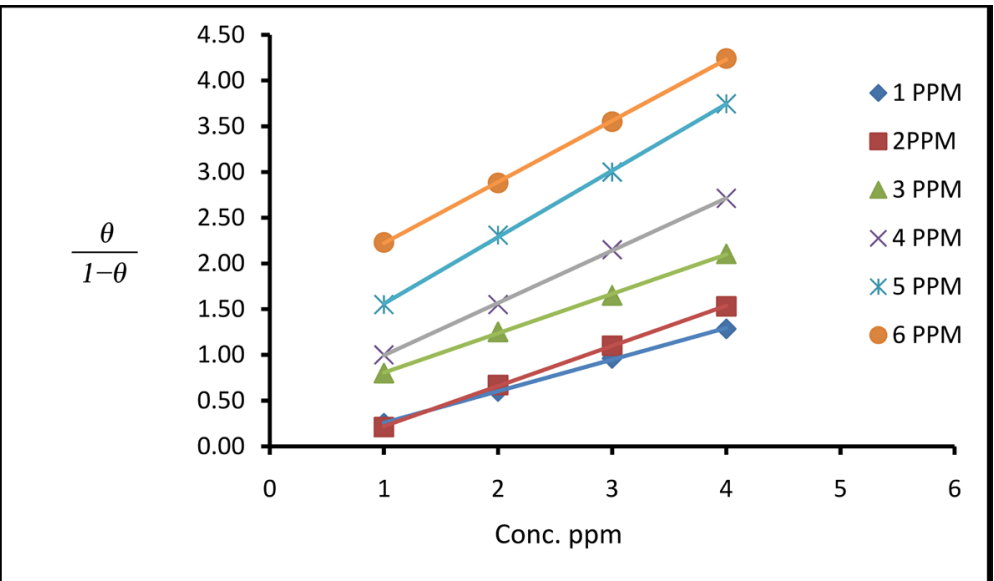

Figure 23. Langmuir s adsorption isotherm plots for the adsorption of GO at different conc. In $1 \mathrm{~N} \mathrm{HCl}$ on carbon steel surface.

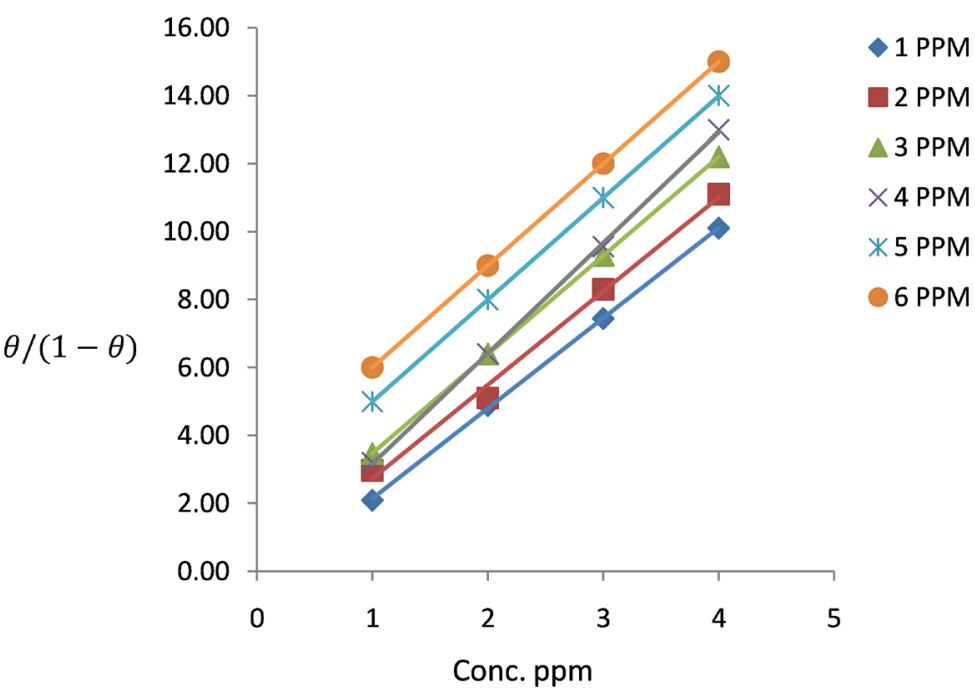

Figure 24. Langmuir s adsorption isotherm plots for the adsorption of GON at different conc. In $1 \mathrm{~N} \mathrm{HCl}$ on carbon steel surface.

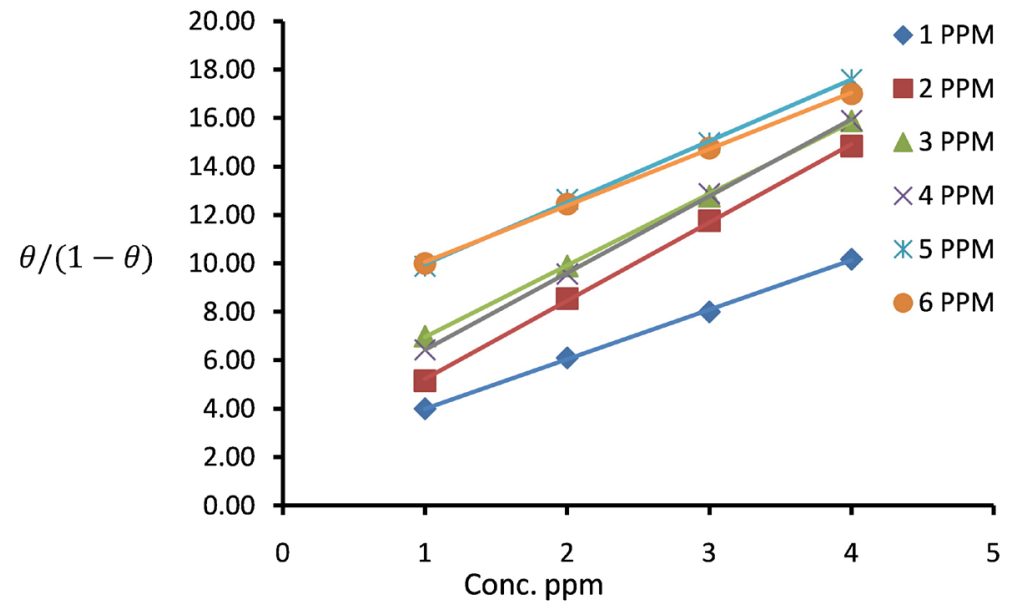

Figure 25. Langmuir s adsorption isotherm plots for the adsorption of GOS at different conc. In $1 \mathrm{~N} \mathrm{HCl}$ on carbon steel surface. 
Table 7. Values of $(\theta / 1-\theta)$, concentration $(\mathrm{ppm})$ and $\mathrm{R}^{2}$ graphene oxide inhibiter at difference temperatures and $(1,6 \mathrm{ppm})$ concentration.

\begin{tabular}{|c|c|c|c|c|c|}
\hline \multirow{2}{*}{ CONC. (ppm) } & \multicolumn{4}{|c|}{$\theta / 1-\theta$} & \multirow{2}{*}{$\mathrm{R}^{2}$} \\
\hline & $298 \mathrm{~K}$ & $308 \mathrm{~K}$ & $318 \mathrm{~K}$ & $328 \mathrm{~K}$ & \\
\hline 1 & 1.28 & 0.96 & 0.60 & 0.25 & 0.9996 \\
\hline 2 & 1.53 & 1.10 & 0.67 & 0.21 & 0.9997 \\
\hline 3 & 2.10 & 1.65 & 1.25 & 0.80 & 0.9994 \\
\hline 4 & 2.71 & 2.15 & 1.55 & 1.00 & 0.9997 \\
\hline 5 & 3.75 & 3.00 & 2.31 & 1.55 & 0.9997 \\
\hline 6 & 4.24 & 3.55 & 2.88 & 2.23 & 0.9998 \\
\hline
\end{tabular}

Table 8. Values of $(\theta / 1-\theta)$, concentration (ppm) and $\mathrm{R}^{2} \mathrm{GON}$ inhibiter at difference temperatures and $(1-6 \mathrm{ppm})$ concentration.

\begin{tabular}{cccccc}
\hline \multirow{2}{*}{ CONC . $(\mathrm{ppm})$} & \multicolumn{3}{c}{$\theta / 1-\theta$} & \multirow{2}{*}{$\mathrm{R}^{2}$} \\
\cline { 2 - 5 } 1 & $298 \mathrm{~K}$ & $308 \mathrm{~K}$ & $318 \mathrm{~K}$ & $328 \mathrm{~K}$ & \\
\cline { 2 - 5 } 2 & 10.10 & 7.44 & 4.88 & 2.09 & 0.9997 \\
3 & 11.10 & 8.30 & 5.10 & 2.97 & 0.9942 \\
4 & 12.20 & 9.30 & 6.40 & 3.47 & 1 \\
5 & 13.00 & 9.56 & 6.40 & 3.20 & 0.9996 \\
6 & 14.00 & 11.00 & 8.00 & 5.00 & 1 \\
\hline
\end{tabular}

Table 9. Values of $(\theta / 1-\theta)$, concentration (ppm) and $\mathrm{R}^{2}$ GOS at difference temperatures and $(1-6 \mathrm{ppm})$ concentration.

\begin{tabular}{cccccc}
\hline \multirow{2}{*}{ CONC. $(\mathrm{ppm})$} & \multicolumn{3}{c}{$\theta / 1-\theta$} & \multirow{2}{*}{$\mathrm{R}^{2}$} \\
\cline { 2 - 5 } 1 & $298 \mathrm{~K}$ & $308 \mathrm{~K}$ & $318 \mathrm{~K}$ & $328 \mathrm{~K}$ & \\
\hline 2 & 10.17 & 8.00 & 6.10 & 4.00 & 0.9994 \\
3 & 14.84 & 11.76 & 8.55 & 5.16 & 0.9995 \\
4 & 15.88 & 12.78 & 9.90 & 7.00 & 0.9997 \\
5 & 15.89 & 12.87 & 9.55 & 6.43 & 0.9997 \\
6 & 17.59 & 14.98 & 12.65 & 9.89 & 0.9998 \\
\hline
\end{tabular}

$$
\Delta \mathrm{G}_{a d s}^{\circ}=-\mathrm{RT} \operatorname{Ln}\left(55.5 K_{a d s}\right)
$$

where $\mathrm{R}$ is the gas constant, $\mathrm{T}$ is the experiment absolute temperature, and theconstant value of 55.5 is the concentration of water in a solution in $\mathrm{mol} \cdot \mathrm{L}^{-1}$. The following equation can be used to calculate a thermodynamic functions [31]

$$
\frac{\Delta \mathrm{G}_{a d s}^{\circ}}{\mathrm{T}}=\frac{\Delta \mathrm{H}_{a d s}^{\circ}}{\mathrm{T}}+K
$$


The variation of $\Delta \mathrm{G}_{a d s}^{\circ} / \mathrm{T}$ with $1 / \mathrm{T}$ gives a straight line with a slope that equals $\Delta \mathrm{H}_{a d s}^{\circ}$ (Figures 26-28). The $\Delta \mathrm{G}_{\text {ads }}^{\circ} / \mathrm{T}$ decreases with $1 / \mathrm{T}$ in a linear manner. The calculated values are shown in Tables 10-12. The adsorption heat could be approximately regarded as the standard adsorption heat under experimental conditions. The negative sign of $\Delta \mathrm{H}^{\circ}$ ads in $1 \mathrm{M} \mathrm{HCl}$ solution indicates that the adsorption of inhibitormolecule is an exothermic process [32]. Then the standard adsorption entropy $\Delta \mathrm{S}_{a d s}^{\circ}$ was obtained using the thermodynamic basic equation:

$$
\Delta \mathrm{G}_{a d s}=\Delta \mathrm{H}_{a d s}-\Delta \mathrm{S}_{a d s}
$$

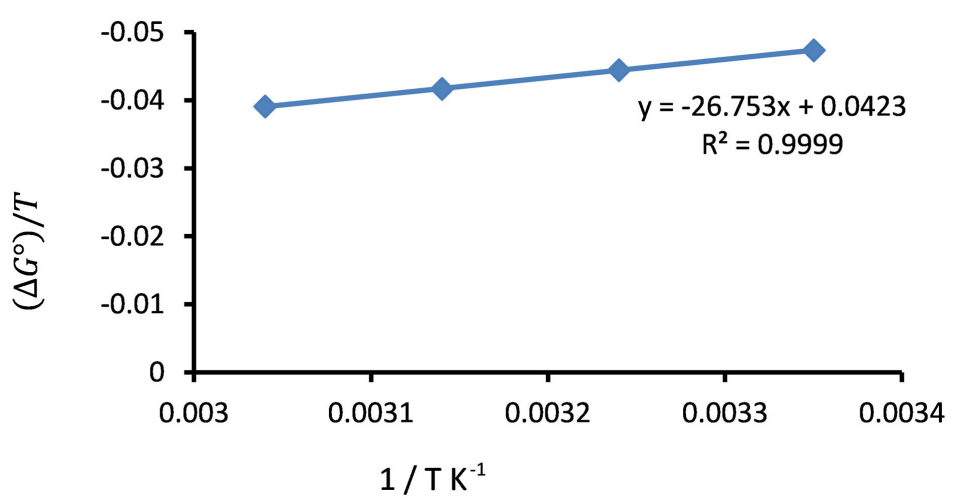

Figure 26. Adsorption isotherm plot for $\Delta \mathrm{G}^{\circ} / \mathrm{T}$ vs $1 / \mathrm{T}$ of $\mathrm{GO}$.

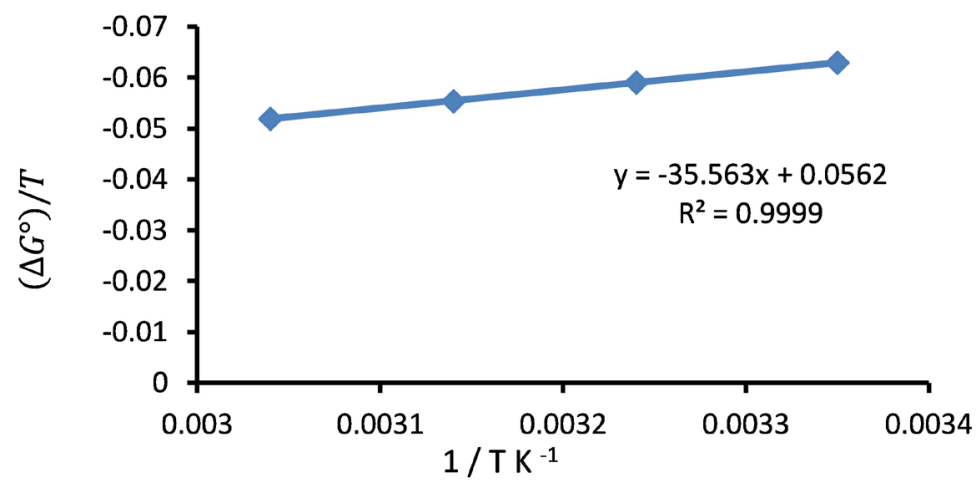

Figure 27. Adsorption isotherm plot for $\Delta \mathrm{G}^{\circ} / \mathrm{T}$ vs $1 / \mathrm{T}$ of GON.

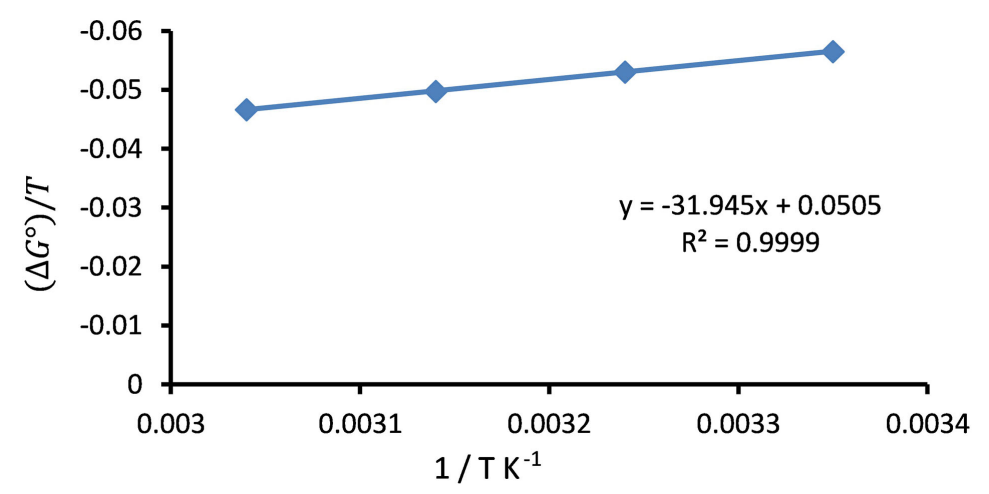

Figure 28. Adsorption isotherm plot for $\Delta \mathrm{G}^{\circ} / \mathrm{T}$ vs $1 / \mathrm{T}$ of GOS. 
Table 10. Thermodynamic parameters for the adsorption of GO in $1 \mathrm{M} \mathrm{HCl}$ on carbon steel surface at different temperatures.

\begin{tabular}{cccc}
\hline Temperture $\mathrm{k}$ & $\begin{array}{c}\Delta \mathrm{G}^{\circ} \\
(\mathrm{KJ} / \mathrm{mol} \cdot \mathrm{K})\end{array}$ & $\Delta \mathrm{H}^{\circ}(\mathrm{KJ} / \mathrm{mol} \cdot \mathrm{K})$ & $\begin{array}{c}\Delta \mathrm{S}^{\circ} \\
(\mathrm{J} / \mathrm{mol} \cdot \mathrm{K})\end{array}$ \\
\hline 298 & -12.82 & -1.30 & 4.30 \\
308 & -13.25 & -1.30 & 4.45 \\
318 & -13.68 & -1.30 & 4.59 \\
328 & -14.11 & -1.30 & 4.74 \\
\hline
\end{tabular}

Table 11. Thermodynamic parameters for the adsorption of GON in $1 \mathrm{~N} \mathrm{HCl}$ on carbon steel surface at different temperatures.

\begin{tabular}{cccc}
\hline Temperture $\mathrm{k}$ & $\begin{array}{c}\Delta \mathrm{G}^{\circ} \\
(\mathrm{KJ} / \mathrm{mol} \cdot \mathrm{K})\end{array}$ & $\Delta \mathrm{H}^{\circ}(\mathrm{KJ} / \mathrm{mol} \cdot \mathrm{K})$ & $\begin{array}{c}\Delta \mathrm{S}^{\circ} \\
(\mathrm{J} / \mathrm{mol} \cdot \mathrm{K})\end{array}$ \\
\hline 298 & -17.05 & -3.98 & 5.75 \\
308 & -17.62 & -3.98 & 5.91 \\
318 & -18.19 & -3.98 & 6.10 \\
328 & -18.76 & -3.98 & 6.30 \\
\hline
\end{tabular}

Table 12. Thermodynamic parameters for the adsorption of GOS in $1 \mathrm{~N} \mathrm{HCl}$ on carbon steel surface at different temperatures.

\begin{tabular}{cccc}
\hline Temperture $\mathrm{k}$ & $\begin{array}{c}\Delta \mathrm{G}^{\circ} \\
(\mathrm{KJ} / \mathrm{mol} \cdot \mathrm{K})\end{array}$ & $\Delta \mathrm{H}^{\circ}(\mathrm{KJ} / \mathrm{mol} \cdot \mathrm{K})$ & $\begin{array}{c}\Delta \mathrm{S}^{\circ} \\
(\mathrm{J} / \mathrm{mol} \cdot \mathrm{K})\end{array}$ \\
\hline 298 & -15.31 & -1.30 & 5.14 \\
308 & -15.83 & -1.30 & 5.31 \\
318 & -16.34 & -1.30 & 5.48 \\
328 & -16.85 & -1.30 & 5.66 \\
\hline
\end{tabular}

\section{Conclusion}

The grapheme oxide GO, GON, and GOS act as corrosion inhibitors of carbon steel in $1 \mathrm{M} \mathrm{HCl}$ solutions. The inhibition efficiency increases with increase in inhibitors concentrations and decreases with raising temperature. The adsorption of the investigated compounds follows the Langmuir's adsorption isotherm. The investigated compounds were mixed type inhibitors. The adsorption of the investigated compound is on carbon steel surface in $\mathrm{HCl}$ solution. Thermodynamic studied $\Delta \mathrm{H}^{*}, \Delta \mathrm{S}^{*}$, and $\mathrm{Ea}^{*}$, indicated to activity of prepared inhibitors and Free energy of adsorption $\Delta \mathrm{G}^{\circ}{ }_{a d s}$ indicated to chemical-physical adsorption.

\section{Conflicts of Interest}

The authors declare no conflicts of interest regarding the publication of this paper. 


\section{References}

[1] Dreyer, D.R., Park, S., Bielawski, C.W. and Ruoff, R.S. (2010) The Chemistry of Graphene Oxide. Chemical Society Reviews, 39, 228, https://doi.org/10.1039/B917103G

[2] Chen, M.-L., Meng, Z.-D., Zhu, L., Choi, J.-G., Park, C.-Y., Lee, S.-C., Hong, D.-S., Lee, J.-G., Jang, W.-K. and Oh, W.-C. (2011) Dispersion Stability of Metal (Oxide)-Graphene Nanofluids with Electrical and Thermal Properties. Science of Advanced Materials, 3, 887-892. https://doi.org/10.1166/sam.2011.1213

[3] Zarrok, H., Al-Deyab, S., Zarrouk A., Salghi, Z., Hammouti, B., Oudda, H., Bouachrine, M. and Bentiss, F. (2012) International Journal of Electrochemical Science, 7, 4047.

[4] Khamis, E., Ameer, M.A, Alandis, N.M. and AL-Senani, G. (2000) Effect of Thiosemicarbazones on Corrosion of Steel in Phosphoric Acid Produced by Wet Process. Corrosion, 56, 127-138. https://doi.org/10.5006/1.3280528

[5] Afia, L., Salghi, R., Benali, O., Jodeh, S., Warad, I., Ebenso, E. and Hammouti, B. (2015) Portugaliae Electrochimica Acta, 33, 137.

[6] de Souza, F.S. and Spinelli, A. (2009) Caffeic Acid as a Green Corrosion Inhibitor for Mild Steel. Corrosion Science, 51 642-649. https://doi.org/10.1016/j.corsci.2008.12.013

[7] Hosseini, S.M.A., Salari, M., Jamalizadeh, E., Khezripoor, S. and Seifi, M. (2010) Inhibition of Mild Steel Corrosion in Sulfuric Acid by Some Newly Synthesized Organic Compounds. Materials Chemistry and Physics, 119, 100-105. https://doi.org/10.1016/j.matchemphys.2009.08.029

[8] Popova, A., Sokolova, E., Raicheva, S. and Christov, M. (2003) AC and DC Study of the Temperature Effect on Mild Steel Corrosion in Acid Media in the Presence of Benzimidazole Derivatives. Corrosion Science, 45, 33-58.

https://doi.org/10.1016/S0010-938X(02)00072-0

[9] Ramesh, S.V. and Adhikari, A.V. (2009) N'-[4-(Diethylamino)Benzylidine]3-\{[8-(Trifluoromethyl) Quinolin-4-Yl]Thio\}Propano Hydrazide) as an Effective Inhibitor of Mild Steel Corrosion in Acid Media. Materials Chemistry and Physics, 115, 618-627. https://doi.org/10.1016/j.matchemphys.2009.01.024

[10] Al Juhaiman, L.A., Mustafa, A.A. and Mekhamer, W.K. (2012) International Journal of Electrochemical Science, 7, 8578.

[11] Alheety, M., Mohammed, M. and Jebour, I. (2016) Synthesis and Haracterization of Novel Nano Dithiocarbamate Complexes Derived From GO-Benzimidazole. Diyala Journal for Pure Science, 16, 108-121.

[12] Shahriary, L. and Athawale, A. (2014) Graphene Oxide Synthesized by Using Modified Hummers Approach. International Journal of Renewable Energy and Environmental Engineering, 2, 58-63.

[13] Paulchamy, B, Arthi, G. and Lignesh, B.D. (2015) A Simple Approach to Stepwise Synthesis of Graphene Oxide Nanomaterial. Nanomedicine \& Nanotechnology, 6, $1-4$,

[14] Yu, Y., Lin, Y., Lin, C., Chan, C. and Hung, Y. (2014) High-Performance Poly Styrene/Grahene-Based Nano Composites with Excellent Anti-Corrosion Properties. Polymer Chemistry, 5, 535-550, https://doi.org/10.1039/C3PY00825H

[15] Al Juhaiman, L.A. (2016) Polyvinyl Pyrrolidone as a Corrosion Inhibitor for Carbon Steel in HCl. International Journal of Electrochemical Science, 11, 2247-2262. 
[16] Chammingkwan, P., Matsushita, K., Taniike, T. and Terano, M. (2016) Enhancement in Mechanical and Electrical Properties of Polypropylene Using Graphene Oxide Grafted with End-Functionalized Polypropylene, Materials, 9, 240. https://doi.org/10.3390/ma9040240

[17] Iram, S. and Hussain, Z. (2015) Covalently Functionalized Graphene Oxide Characterization and Its Electrochemical Performance. International Journal of Electrochemistry Science, 10, 9475-9487.

[18] Monshi, A., Foroughi, M., Monshi, R. and Modified, M.R. (2012) Scherrer Equation to Estimate More Accurately Nano-Crystallite Size Using XRD. World Journal of Nano Science and Engineering, 2, 154-160. https://doi.org/10.4236/wjnse.2012.23020

[19] Theivasanthi, T. and Alagar, M. (2011) Nano Sized Copper Particles by Electrolytic Synthesis and Characterizations. International Journal of the Physical Sciences, 6, 3662-3671.

[20] Meng, F., Ishidab, H. and Liu, X. (2014) Introduction of Benzoxazine onto the Grapheme Oxide Surface by Click Chemistry and the Properties of Graphene Oxide Reinforced Polybenzoxazine Nanohybrids. RSC Advance, 4, 9471-9475. https://doi.org/10.1039/c3ra47345g

[21] Lo, C., Zhu, D. and Jiang, H. (2011) An Infrared-Light Responsive Graphene-Oxide Incorporatedpoly (N-Isopropylacrylamide) Hydrogel Nanocomposite. Soft Matter, 7, 5604-5609. https://doi.org/10.1039/c1sm00011j

[22] Serrar, H., Laroujb, M., Gazc, H.L., Benzekri, Z., Zarguil, A., Essebaai, H., Boukhris, Ouddab, S.H., Salghi, R., Hassikou, A. and Souizi, A. (2018) Experimental and Theoretical Studies of the Corrosion Inhibition of 4-amino-2-(4-chlorophenyl)-8(2, 3-dimethoxyphenyl)-6-oxo-2,6-dihydropyrimido [2,1-b] [1,3] thiazine-3,7 dicarbonitrile on Carbon Steel in a $1.0 \mathrm{M} \mathrm{HCl}$ Solution. Portugaliae Electrochimica Acta, $36,35-52$.

[23] Elkhotfi, Y., Forsal, I., Rakib, E.M. and Mernari, B. (2018) The Inhibition Action of Essential Oil of J. Juniperus Phoenicea on the Corrosion of Mild Steel in Acidic Media. Portugaliae Electrochimica Acta, 36, 77-87.

[24] Abdallah, M., Fouad, A., Shama, S. and Afifi, E. (2008) Azodyes as Corrosion Inhibitors for Dissolution of C-Steel in $\mathrm{HCl}$ Solution. African Pure Application Chemistry, 2, 83-91.

[25] Al-Sawaad, H.Z.M. (2013) Evaluation of the Ceftriaxone as Corrosion Inhibitor for Carbon Steel Alloy in $0.5 \mathrm{M}$ of Hydrochloric Acid. International Journal of Electrochemical Science, 8, 3105-3120.

[26] Bhat, J. and Alva, V. (2009) Corrosion Inhibition of Aluminium by 2-Chloronicotinic Acid in $\mathrm{HCl}$ Medium. Indian Journal of Chemical Technology, 16, 228-233.

[27] Al Juhaiman, L.A. (2016) Polyvinyl Pyrrolidone as a Corrosion Inhibitor for Carbon Steel in HCl. International Journal of Electrochemical Science, 11, 2247-2262.

[28] Dahmani, M., Et-Touhami, A., Al-Deyab, S.S., Hammouti, B. and Bouyanzer, A. (2010) Corrosion Inhibition of C38 Steel in 1M HCl: A Comparative Study of Black Pepper Extract and Its Isolated Piperine. International Journal of Electrochemical Science, 5, 1060-1069.

[29] Abdullah, A. (2008) Adsorption Isotherm of Some Triazoles as Corrosion Inhibitors of Mild Steel in Acids. AL-Fatih, 32.

[30] Abdallah EL, M., Helal, E. and Fouda, A. (2006) Corrosion Science, 84, 1639.

[31] El-Etre, A.Y. (2007) Inhibition of Acid Corrosion of Carbon Steel Using Aqeous 
Extract of Olive Leaves. Journal of Colloid and Interface Science, 314, 578-583. https://doi.org/10.1016/j.jcis.2007.05.077

[32] AI-Juaid, S. (2007) Mono Azo Dyes Compounds as Corrosion Inhibitors for Dissolution of Aluminium in Sodium Hydroxide Solutions. Portugaliae Electrochimica Acta, 25, 363-373. 